\title{
Understanding Hadley Cell Expansion versus Contraction: Insights from Simplified Models and Implications for Recent Observations
}

\author{
NEIL F. TANDON \\ Department of Applied Physics and Applied Mathematics, Columbia University, New York, New York \\ EDWIN P. GERBER \\ Center for Atmosphere Ocean Science, Courant Institute of Mathematical Sciences, New York University, \\ New York, New York
}

Adam H. Sobel And Lorenzo M. Polvani

Department of Applied Physics and Applied Mathematics, and Department of Earth and Environmental Sciences, Columbia University, New York, New York

(Manuscript received 7 August 2012, in final form 13 December 2012)

\begin{abstract}
This study seeks a deeper understanding of the causes of Hadley Cell (HC) expansion, as projected under global warming, and $\mathrm{HC}$ contraction, as observed under El Niño. Using an idealized general circulation model, the authors show that a thermal forcing applied to a narrow region around the equator produces "El Niño-like" HC contraction, while a forcing with wider meridional extent produces "global warming-like" $\mathrm{HC}$ expansion. These circulation responses are sensitive primarily to the thermal forcing's meridional structure and are less sensitive to its vertical structure. If the thermal forcing is confined to the midlatitudes, the amount of $\mathrm{HC}$ expansion is more than three times that of a forcing of comparable amplitude that is spread over the tropics. This finding may be relevant to recently observed trends of rapid tropical widening.

The shift of the HC edge is explained using a very simple model in which the transformed Eulerian mean (TEM) circulation acts to diffuse heat meridionally. In this context, the HC edge is defined as the downward maximum of residual vertical velocity in the upper troposphere $\bar{\omega}_{\max }^{*}$; this corresponds well with the conventional Eulerian definition of the $\mathrm{HC}$ edge. In response to a positive thermal forcing, there is anomalous diabatic cooling, and hence anomalous TEM descent, on the poleward flank of the thermal forcing. This causes the $\mathrm{HC}$ edge $\left(\bar{\omega}_{\max }^{*}\right)$ to shift toward the descending anomaly, so that a narrow forcing causes $\mathrm{HC}$ contraction and a wide forcing causes $\mathrm{HC}$ expansion.
\end{abstract}

\section{Introduction}

How does the large-scale atmospheric circulation respond to changing temperatures? This is an important question in climate change research, and it has motivated many past studies. These include numerous idealized modeling experiments examining the circulation's response to thermal forcings in the stratosphere (e.g., Polvani and Kushner 2002; Haigh et al. 2005; Gerber and Polvani 2009; Tandon et al. 2011) as well as the troposphere (e.g., Son and Lee 2005; Kang et al. 2009; Butler

Corresponding author address: Neil F. Tandon, Columbia University, 500 W 120 St., Room 200 Mudd, New York, NY 10027.

E-mail:nft2104@columbia.edu et al. 2010; Wang et al. 2012). The understanding of circulation changes over the long term is often informed by the study of short-term activity, such as stratospheric sudden warmings (e.g., Gerber et al. 2009) and volcanic eruptions (e.g., Soden et al. 2002).

In particular, the study of El Niño-Southern Oscillation (ENSO) has greatly aided our understanding of circulation change in the climate context. Using a general circulation model (GCM) with forced sea surface temperature (SST), Seager et al. (2003) examined the dynamics of the El Niño-driven circulation response in great detail. They found that the short-term response to El Niño SST anomalies resembles the steady-state response to a persistent SST increase in the deep tropics. This makes for a natural comparison between the 
El Niño circulation response and the response to the long-term increase of greenhouse gases, commonly termed the "global warming" response.

Under global warming, most coupled models produce enhanced warming of SSTs in the eastern tropical Pacific (e.g., DiNezio et al. 2009), a pattern resembling El Niño. This led to the hypothesis that the circulation response to global warming might resemble the circulation response to El Niño. Lu et al. (2008) tested this by performing a detailed analysis of output from coupled GCMs. They found that the circulation response due to global warming is in many respects qualitatively opposite to that of El Niño. Specifically, global warming produces expansion and weakening of the Hadley Cell (HC), while El Niño produces contraction and strengthening of the HC. Also, global warming produces a poleward shift of the midlatitude jets, while El Niño produces an equatorward shift. This contrast is intriguing because both El Niño and global warming produce substantial warming of the tropical troposphere (Lu et al. 2008). This means that seemingly subtle alterations to the structure of a thermal forcing can have a dramatic effect on the circulation response. It is this sensitivity that is the focus of this paper.

The results of earlier studies point to a key factor behind this sensitivity. Chang (1995) and Son and Lee (2005), using idealized dry GCM, showed that a thermal forcing applied to a narrow region around the equator in the lower troposphere produces an equatorward shift of the midlatitude jets. This contrasts with the findings of Butler et al. (2010) and Wang et al. (2012), who found that heating with wider meridional extent in the tropical upper troposphere produces a poleward shift of the jets. Furthermore, Chen et al. (2010) have shown that changes to the meridional structure of the SST forcing in an atmosphere-only GCM produces a transition from an El Niño-like circulation response to a global warminglike response. Altogether, these earlier studies suggest that the contrast between the global warming and the El Niño circulation responses may be attributable to either the meridional extent or the vertical structure of the thermal forcing.

This provides the inspiration for the present study. Specifically, we take an idealized GCM and apply thermal forcings of varying meridional width centered at the equator (section 2). We show that narrow thermal forcings produce El Niño-like HC contraction, while wider thermal forcings produce global warming-like $\mathrm{HC}$ expansion. We also show that changes in the vertical structure of the forcing have a relatively minor effect on the circulation response. The HC turns out to be particularly sensitive to warming in the midlatitudes, a finding which may be relevant in light of recent observations. In addition, we construct a simple diffusive model of the transformed Eulerian mean (TEM) circulation to explain the transition from $\mathrm{HC}$ contraction to $\mathrm{HC}$ expansion (section 3 ).

Earlier idealized modeling studies have focused either on the El Niño circulation response alone (e.g., Robinson 2002; Seager et al. 2003) or on the global warming response alone (e.g., Kidston et al. 2010; Levine and Schneider 2011; Rivière 2011). Thus, it has remained unclear how the mechanisms driving the El Niño- and global warminglike responses fit into the same physical framework. By studying both phenomena together, we can develop a more comprehensive understanding of what drives changes in the tropospheric circulation.

\section{Experiments with an idealized GCM}

\section{a. Method}

Our idealized GCM is a dynamical core forced with highly simplified radiation and convection schemes. This GCM is nearly identical to that used in Tandon et al. (2011), and we provide complete details in the appendix. In the GCM's radiation scheme, temperatures are linearly relaxed to a prescribed equilibrium profile which mimics a gray atmosphere (Schneider 2004; Schneider and Walker 2006). When a column becomes statically unstable, the temperature in the column is relaxed to a moist adiabatic profile that conserves enthalpy (Schneider and Walker 2006). This convection scheme compensates to an extent for the lack of explicit moisture in the model. The lapse rate of the convective equilibrium profile is a prescribed parameter, and we experiment with perturbing this parameter, as described below. Compared to dry models that use the Held and Suarez (1994) forcings (e.g., Son and Lee 2005; Butler et al. 2010, 2011; Wang et al. 2012), the model we use produces a climatology with more realistic stratification and tropopause height in the tropics (Tandon et al. 2011).

We run the GCM in a perpetual equinox configuration with hemispherically symmetric radiative forcing. All integrations are performed at spectral resolution T42 with 40 vertical levels (see the appendix for additional details). We have verified that all of our key results are robust to doubling of either the horizontal or vertical resolution.

In each integration, we impose an additional thermal forcing consisting of 1) warming of lower-tropospheric temperatures, mimicking an increase in longwave opacity, and 2) a decrease of the convective equilibrium lapse rate. This lapse-rate perturbation mimics the lapserate feedback in a moist atmosphere, which reduces warming near the surface and amplifies it aloft. The lower-tropospheric thermal forcing $\tilde{Q}$ takes the form of 
a potential temperature tendency that is added to the heat equation. Specifically,

$$
\tilde{Q}\left(\phi, p ; \phi_{w} ; \alpha\right)=\frac{\alpha \tilde{Q}_{0}}{\phi_{w}} e^{-\left(\phi / \phi_{w}\right)^{2}}\left(\frac{p}{p_{0}}\right)^{2.4},
$$

where $\phi$ is latitude, $p$ is pressure, $\tilde{Q}_{0}=18 \mathrm{~K} \mathrm{day}^{-1} \times 1^{\circ}$ latitude, and $p_{0}=1000 \mathrm{hPa}$. The meridional $e$-folding width of the thermal forcing is controlled by the parameter $\phi_{w}$, and we refer to this simply as the "width" of the thermal forcing. The factor of $\tilde{Q}_{0} / \phi_{w}$ serves to keep the area integral of $\tilde{Q}$ constant as $\phi_{w}$ is varied. The value of $\tilde{Q}_{0}$ has been chosen so that, for all thermal forcings, the globally averaged temperature increase at the lowest model level is $2-3 \mathrm{~K}$. The factor $\alpha$ is used to scale the relative amplitude of the thermal forcing; we set $\alpha=1$ in all cases unless stated otherwise.

In addition to this lower-tropospheric forcing, we also perturb the lapse rate of the model's convective equilibrium profile. This perturbation takes the form

$$
\tilde{\Gamma}\left(\phi ; \phi_{w}\right)=\tilde{\Gamma}_{0} e^{-\left(\phi / \phi_{w}\right)^{2}},
$$

where $\tilde{\Gamma}_{0}=-0.2 \mathrm{~K} \mathrm{~km}^{-1}$ unless stated otherwise. Note that the parameter $\phi_{w}$ appears in both (1) and (2), so this single parameter controls the meridional extent of both the lower-tropospheric forcing and the lapse-rate forcing.

We have selected thermal forcings with a range of $\phi_{w}$ values to examine the El Niño- and global warming-like responses, as well as the transition between them. We will refer to these integrations using the following labels.

- Phi5, with $\phi_{w}=5^{\circ}$, is a narrow El Niño-like perturbation with peak thermal forcing between $-5^{\circ}$ and $5^{\circ}$ latitude. This forcing is shown in Fig. 1a.

- Phi35, with $\phi_{w}=35^{\circ}$, is a wider global warming-like thermal forcing (Fig. 1b).

- Phi15 $\left(\phi_{w}=15^{\circ}\right)$, Phi20 $\left(\phi_{w}=20^{\circ}\right)$, and Phi25 $\left(\phi_{w}=\right.$ $25^{\circ}$ ) are intermediate cases, meant to examine the transition from $\mathrm{HC}$ contraction to $\mathrm{HC}$ expansion as well as the linearity of the circulation responses.

- Phi35-20 is a special case in which we confine the lowertropospheric forcing between $20^{\circ}$ and $35^{\circ}$ latitude in each hemisphere, while applying a lapse-rate perturbation between $-35^{\circ}$ and $35^{\circ}$ latitude (Fig. 1c). In the notation of (1) and (2), the lower-tropospheric forcing is

$$
\frac{\phi_{w 2} \tilde{Q}\left(\phi, p ; \phi_{w 2} ; \alpha\right)-\phi_{w 1} \tilde{Q}\left(\phi, p ; \phi_{w 1} ; \alpha\right)}{\phi_{w 2}-\phi_{w 1}}
$$

and the lapse-rate perturbation is $\tilde{\Gamma}\left(\phi ; \phi_{w 2}\right)$, where $\phi_{w 1}=20^{\circ}$ and $\phi_{w 2}=35^{\circ}$. This is qualitatively the same (a) Phi5

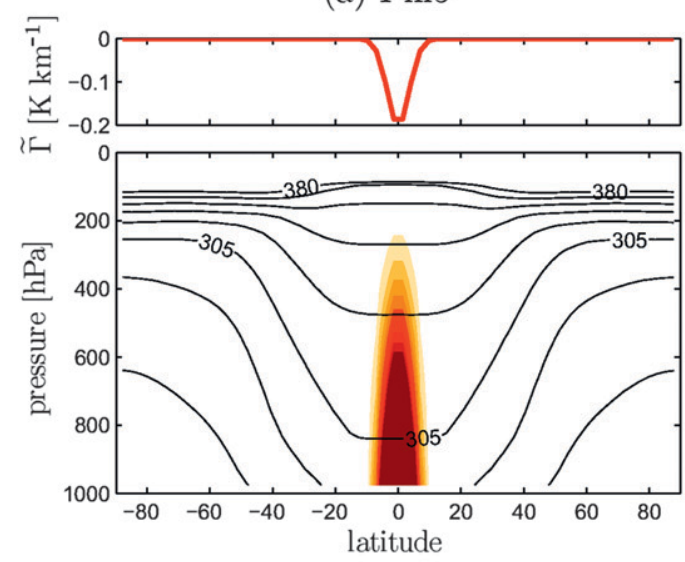

(b) Phi35

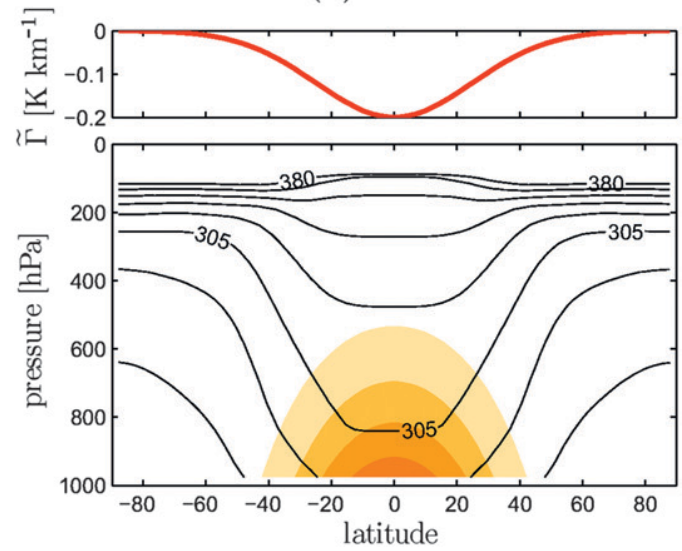

(c) Phi35-20

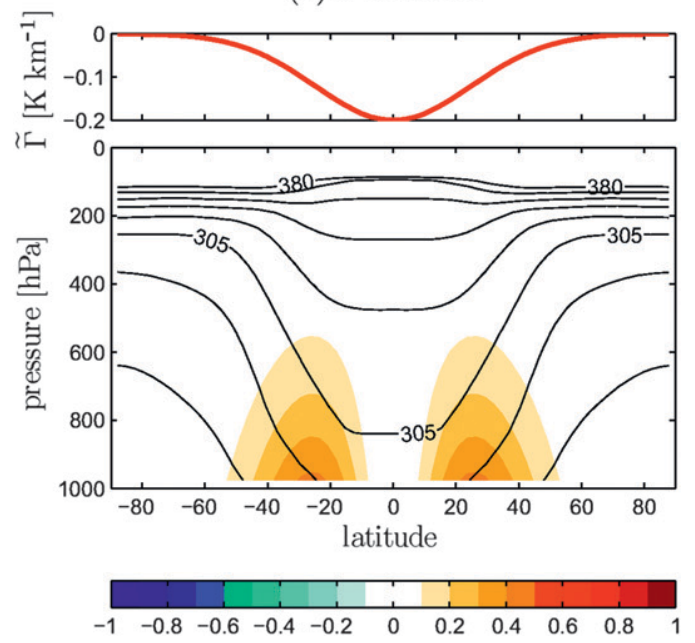

FIG. 1. (a)-(c) Thermal forcings applied in our idealized GCM integrations. Color shading shows the lower-tropospheric thermal forcings with shading interval $0.1 \mathrm{~K} \mathrm{day}^{-1}$. Black contours show potential temperature of the control integration, with contour interval of $15 \mathrm{~K}$ and contours above $380 \mathrm{~K}$ omitted. Red curves are the perturbations of the convective equilibrium lapse rate, meant to mimic the lapse-rate feedback. 
Phi5

(a) $\Delta T[\mathrm{~K}]$

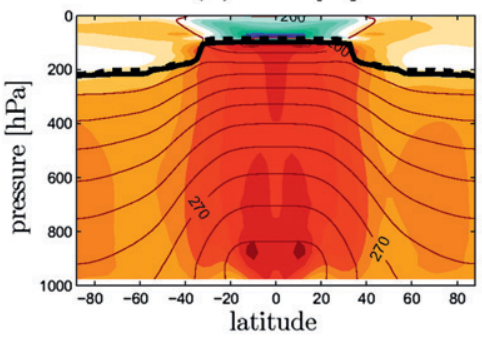

(b) $\Delta u\left[\mathrm{~m} \mathrm{~s}^{-1}\right]$

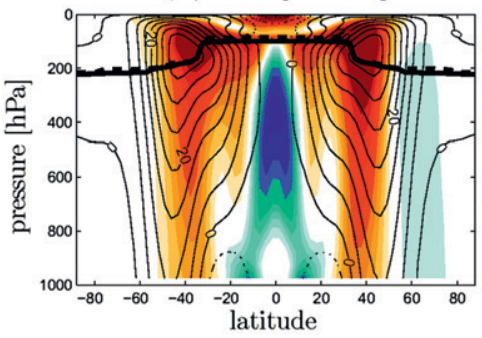

(c) $\Delta \Psi\left[10^{9} \mathrm{~kg} \mathrm{~s}^{-1}\right]$

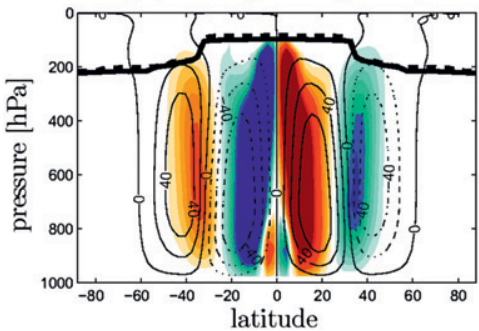

Phi35

(d) $\Delta T[\mathrm{~K}]$

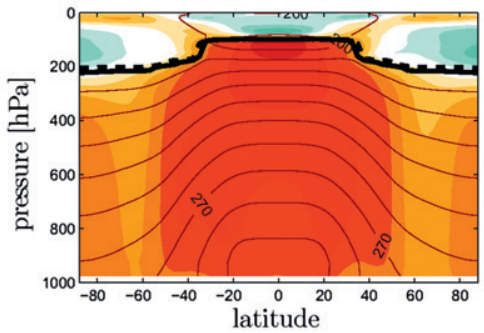

(e) $\Delta u\left[\mathrm{~m} \mathrm{~s}^{-1}\right]$

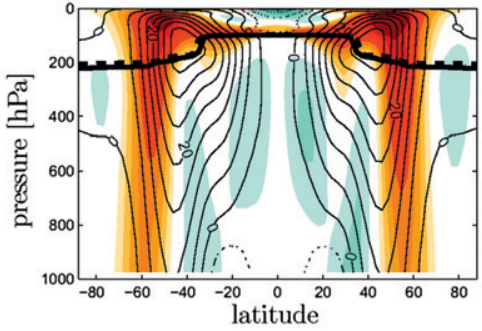

(f) $\Delta \Psi\left[10^{9} \mathrm{~kg} \mathrm{~s}^{-1}\right]$

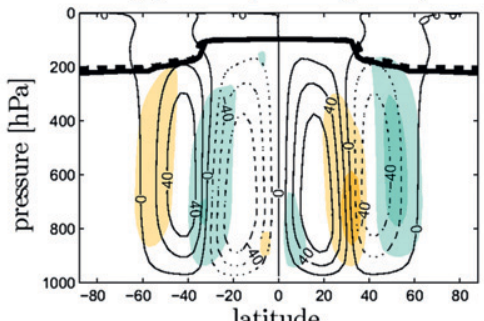

Phi35-20

(g) $\Delta T[\mathrm{~K}]$

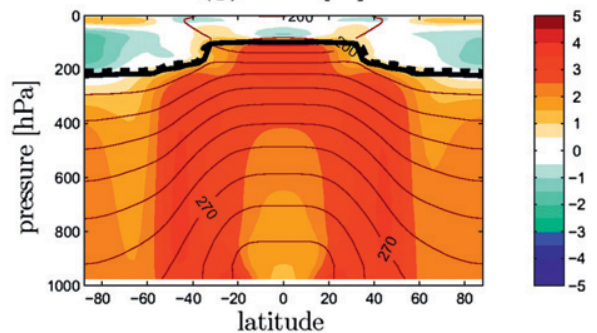

(h) $\Delta u\left[\mathrm{~m} \mathrm{~s}^{-1}\right]$

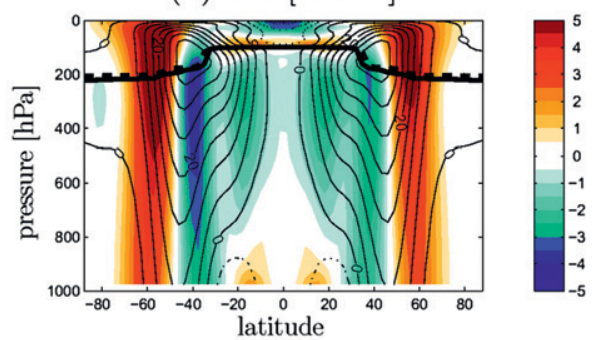

(i) $\Delta \Psi\left[10^{9} \mathrm{~kg} \mathrm{~s}^{-1}\right]$

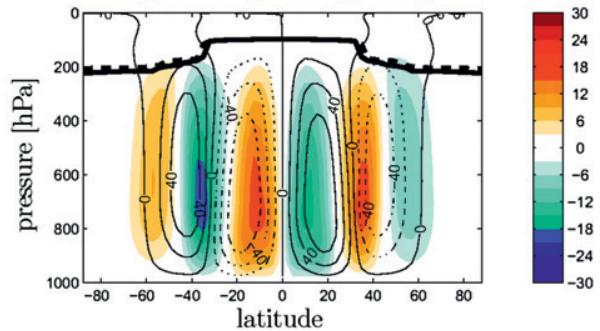

FIG. 2. The steady-state responses to the thermal forcings indicated at the top of each column. Color shading shows: (a),(d),(g) the difference between the climatologies of the forced and control integrations for temperature; (b),(e),(h) zonal wind; and (c),(f),(i) meridional mass streamfunction. Thin black contours show the climatology of the control integration, with contour intervals of $10 \mathrm{~K}$ for temperature in the top row; $5 \mathrm{~m} \mathrm{~s}^{-1}$ for zonal wind in the middle row with negative contours dashed; and $20 \times 10^{9} \mathrm{~kg} \mathrm{~s}{ }^{-1}$ for the meridional mass streamfunction in the bottom row with negative contours dashed. Positive streamfunction values indicate clockwise motion and negative values indicate counterclockwise motion. The solid, thick black contour is the thermal tropopause of the control integration. The dashed, thick, black contour is the tropopause of the forced integration.

as the Phi35 forcing, but with the tropical lower tropospheric portion removed.

- Forcings with the additional LT label (e.g., Phi5 $5_{\mathrm{LT}}$, Phi35 $\mathrm{LT}$, etc.) are identical to the standard forcings above, except the thermal forcing is applied only in the lower troposphere without any lapse-rate forcing (i.e., $\tilde{\Gamma}_{0}=0$ ). This is meant to test the sensitivity of the circulation response to the change in the lapse rate.

- Forcings with the additional UT label (e.g., Phi5 $5_{\mathrm{UT}}$, Phi35 $\mathrm{UT}$, etc.) are identical to the standard forcings above, except the decrease in the lapse rate is doubled, that is, $\tilde{\Gamma}_{0}=-0.4 \mathrm{~K} \mathrm{~km}^{-1}$. This is comparable to the lapse-rate change in the upper troposphere in coupled GCM simulations of global warming (Lorenz and DeWeaver 2007, Fig. 2b).
For each thermal forcing, we start the model from rest and integrate for a total of 4000 days, which is sufficient to obtain a statistically stationary climatology. To compute all climatological fields, we discard the first 200 days as spinup and time average the rest. To obtain the "response" of the model, we subtract the climatology of a control integration in which no thermal forcing is applied (i.e., $\tilde{Q}=0$ and $\tilde{\Gamma}=0$ ). Since there is no topography in this model and all forcings are hemispherically symmetric, the model responses should be hemispherically symmetric; any small asymmetry that remains is due to sampling error.

\section{b. Results}

Figure 2 shows the model responses to the three thermal forcings shown in Fig. 1; these forcings have the 
same area integral and vary only in their meridional structure. Figure 2, first column, shows the response to the Phi5 forcing, which is confined to a narrow band around the equator. The peak warming (Fig. 2a, shading) extends to the top of the troposphere because we have imposed a decrease of the convective equilibrium lapse rate in addition to the lower tropospheric thermal forcing. In the midlatitudes, there is a local minimum in warming. There is also a slight rise in global tropopause height (thick dashed contour), where the tropopause is defined using the standard lapse-rate criterion (World Meteorological Organization 1957).

The Phi5 zonal wind response (Fig. 2b, shading) shows eastward acceleration on the equatorward flanks of the midlatitude jets, indicating equatorward shifts of the jets. Near the equator, there is strong westward acceleration. Figure $2 \mathrm{c}$ shows the response of the meridional overturning streamfunction $\Psi$. [see Peixoto and Oort (1992), section 7.4.3 for the definition.] In the Northern Hemisphere $(\mathrm{NH})$, there is anomalous clockwise motion in the middle and upper portions of the $\mathrm{HC}$, indicating a strengthening and deepening of the HC. There is also a counterclockwise anomaly at the poleward edge of the $\mathrm{HC}$, indicating equatorward contraction of the $\mathrm{HC}$ and anomalous ascent in the midlatitudes. This anomalous ascent coincides with the midlatitude minimum in the temperature response (Fig. 2a). At the equator, $\Psi$ decreases near the surface and increases at higher levels, indicating a decrease in vertical velocity near the surface and an increase aloft. Note that the response of $\Psi$ in the Southern Hemisphere (SH) has the opposite sign, but the physical interpretation is identical. So overall, the Phi5 response resembles El Niño's circulation response of comprehensive models (Seager et al. 2003; Lu et al. 2008). One discrepancy is that El Niño's temperature response in comprehensive models shows cooling in the midlatitudes, which is not reproduced in our model (Fig. 2a), but the circulation responses are in agreement. Another discrepancy is that comprehensive models produce much less westward acceleration at the equator, even though the eastward anomalies in the midlatitudes are of comparable magnitude (cf. Lu et al. 2008).

We next consider the response when the thermal forcing is widened meridionally. This is captured by the results of the Phi35 integration, shown in Fig. 2, second column. Because of the wider thermal forcing, the peak temperature response (Fig. 2d) is spread wider meridionally than for Phi5, and there is a clear contrast between warming in the tropical lower troposphere and the amplified warming aloft. There is also dynamically induced cooling in the extratropical stratosphere, similar to that found in other idealized modeling studies (Butler et al. 2010, 2011; Wang et al. 2012). As in the
Phi5 integration, there is a slight increase in global tropopause height. The zonal wind response (Fig. 2e) shows a clear dipole of westward-eastward acceleration flanking the jet, indicating a poleward shift of the jet. The meridional streamfunction (Fig. 2f) shows expansion of the HCs and poleward shifts of the Ferrel Cells, although the changes in $\Psi$ are substantially lower in magnitude than for Phi5. In short, the circulation response of Phi35 resembles the global warming response of comprehensive models (e.g., Yin 2005; Miller et al. 2006; Gastineau et al. 2008; Wu et al. 2011), and it is in most respects qualitatively opposite to the El Niño-like response of Phi5.

Note, we are not claiming that the Phi5 and Phi35 forcings are actually equivalent to the heating produced by El Niño and increased well-mixed greenhouse gases. Of course, with an actual El Niño, there is no simple external forcing: the changes in diabatic heating are internally determined by feedbacks between the atmosphere and the ocean. Our focus here is on understanding the circulation responses to various external thermal forcings, as a key step toward understanding circulation change in more realistic models and observations. In this regard, simple thermal forcings like Phi5 and Phi35 are sufficient to produce circulation responses resembling those produced under El Niño and global warming, respectively.

Also worth noting is that even though the Phi35 forcing is spatially confined, the temperature response shows substantial warming throughout the troposphere (Fig. 2d). (Indeed, this is true of all the thermal forcings considered in this paper.) This contrasts with the temperature responses of Butler et al. $(2010,2011)$, which are more spatially confined. Unlike the model used in Butler et al. (2010, 2011), our model uses a statically unstable radiative equilibrium profile and parameterized convection, but precisely how these produce differences in the temperature responses requires further work.

The fact that the circulation responses of Phi5 and Phi35 are opposite in sign leads to another question: is the system linearly additive? That is, if we apply a thermal forcing like Phi35, but remove the portion near the equator, do we actually obtain more $\mathrm{HC}$ expansion compared to Phi35? We address this question more rigorously below, but as a first crude test, we consider the Phi35-20 forcing. This forcing is qualitatively the same as Phi35, except that the forcing amplitude approaches zero between $-20^{\circ}$ and $20^{\circ}$ latitude in the lower troposphere (Fig. 1c). The temperature response (Fig. 2g) shows peak warming in the subtropics and midlatitudes, along with enhanced warming in the tropical upper troposphere. The zonal wind response (Fig. 2h) is of 
(a)

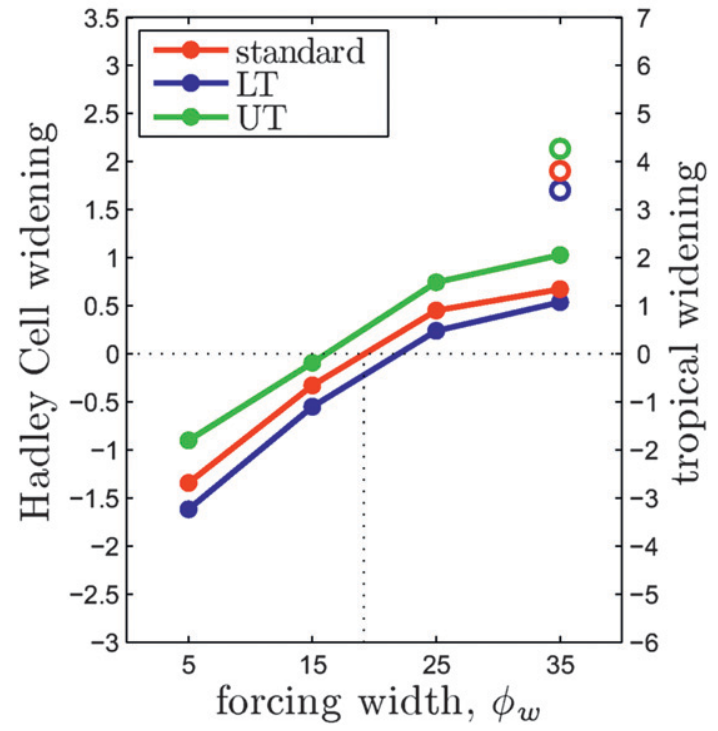

(b)

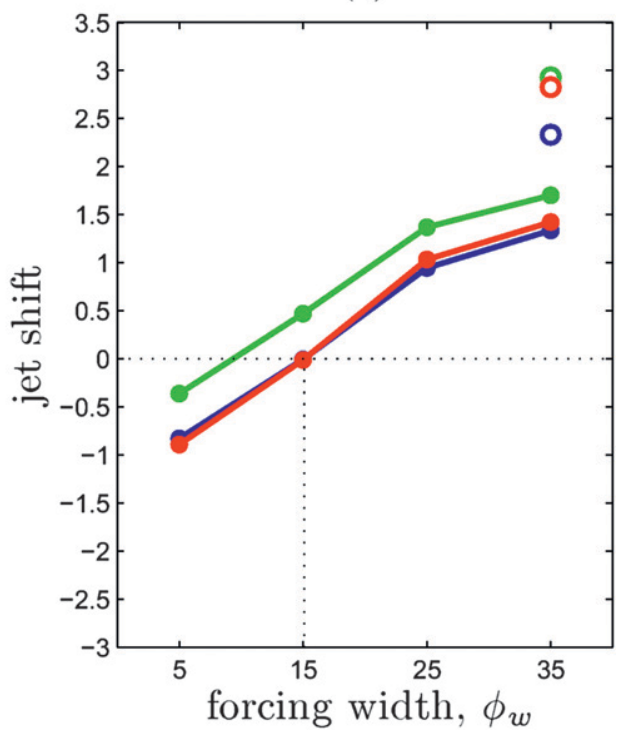

FIG. 3. Changes in circulation metrics as functions of meridional width of the thermal forcing $\phi_{w}$. Red circles refer to standard integrations with both lower tropospheric and lapse-rate forcings. Blue circles refer to integrations with only lower-tropospheric forcing and no lapse-rate forcing. Green circles refer to integrations in which the lapse-rate perturbation is increased. Empty circles indicate results from the Phi35-20 integrations. (a) The shift of the HC edge, defined using the standard $\Psi_{500}$ metric (see text). The right-hand $y$ axis multiplies the shift of the HC edge by two to measure the total tropical widening. (b) The shift of the midlatitude jet. Positive values on the $y$ axis indicate poleward shifts. Vertical dotted lines mark the zero crossings for the standard integrations. Northern and Southern Hemisphere values have been averaged together.

substantially larger magnitude than in Phi35 (Fig. 2e), indicating a larger poleward shift of the jets. The zonal wind anomalies are also more vertically uniform than those of Phi35. The response of the meridional streamfunction (Fig. 2i) is also larger than that of Phi35 (Fig. 2f), indicating greater expansion and weakening of the HC. Thus overall, the circulation response of Phi35-20 qualitatively resembles the global warming-like response of Phi35, but quantitatively the Phi35-20 response is greatly amplified.

Beyond these illustrative examples, we have also performed a sweep of the parameter $\phi_{w}$, which controls the meridional width of the thermal forcing. Figure 3, red circles, shows the associated shifts of the $\mathrm{HC}$ edge (Fig. 3a) and the midlatitude eddy-driven jet (Fig. 3b). The midlatitude jet is located by finding the latitude of maximum zonal wind at the lowest model level. We locate the $\mathrm{HC}$ edge using the standard $\Psi_{500}$ metric - that is, moving poleward from the subtropical maximum of $|\Psi|$, we find the first zero crossing of $\Psi$ at $500 \mathrm{hPa}$. Note that, because of the hemispheric symmetry of our model, a poleward shift of the $\mathrm{HC}$ edge implies a widening of the $\mathrm{HC}$, and multiplying this widening by two gives the overall widening of the tropical belt (cf. Seidel et al. 2008; Johanson and Fu 2009; Davis and Rosenlof 2012).
Figure 3 shows that there is a smooth transition from equatorward jet shift and $\mathrm{HC}$ contraction to poleward jet shift and HC expansion. Interestingly, the zero crossings (vertical dotted lines) are not the same for the two metrics, showing slight HC contraction still occurs even when there is no jet shift. At these zero crossings, there is still a circulation response, but the position of the anomalies with respect to the climatology is such that no shift occurs. For example, in the Phi15 case (not shown), there is eastward acceleration centered precisely over the jet, whereas for other values of $\phi_{w}$, the acceleration occurs more on the flanks of the jet. Figure 3 also shows the large quantitative difference between the Phi35-20 integration and the other integrations. Comparing the empty red circles with the other points, one sees that Phi35-20 produces a factor of four increase in $\mathrm{HC}$ expansion (Fig. 3a) and a factor of two increase in jet shift (Fig. 3b).

We have found that the amount of HC expansion and jet shift has relatively little sensitivity to the change in the lapse rate. To demonstrate this, we have performed a series of integrations in which the thermal forcings have identical meridional structures to those in Fig. 1, but without any changes in the lapse rate. We mark these integrations with the additional label "LT," and the results are plotted in blue in Fig. 3. Removing the lapse-rate 
Phi35 $5_{\mathrm{LT}}$

(a) $\Delta T[\mathrm{~K}]$

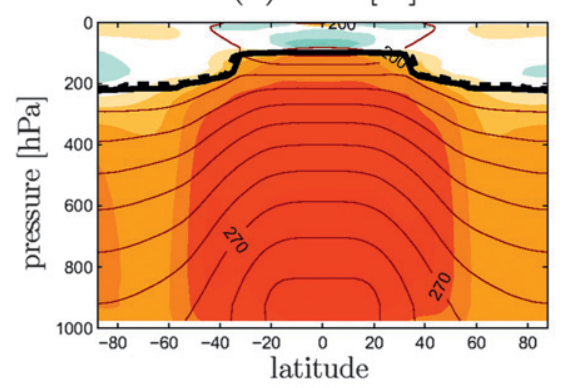

(b) $\Delta u\left[\mathrm{~m} \mathrm{~s}^{-1}\right]$

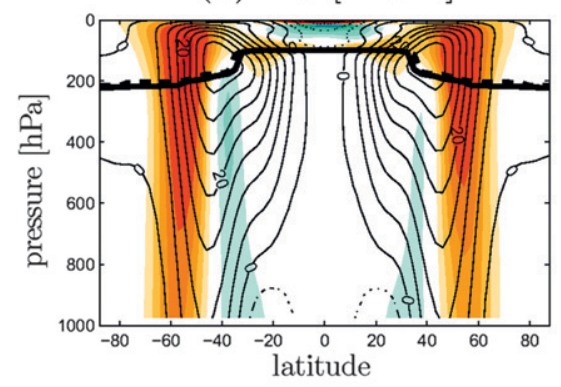

(c) $\Delta \Psi\left[10^{9} \mathrm{~kg} \mathrm{~s}^{-1}\right]$

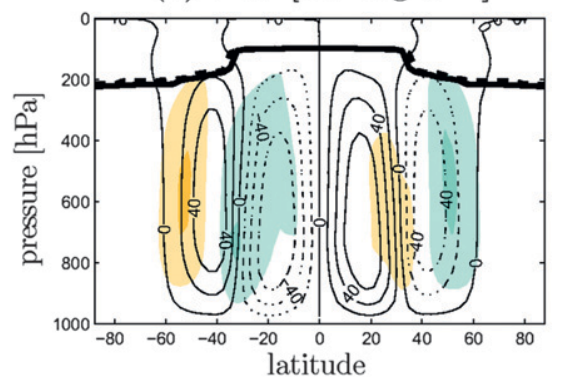

Phi35 $5_{\text {UT }}$

(d) $\Delta T[\mathrm{~K}]$

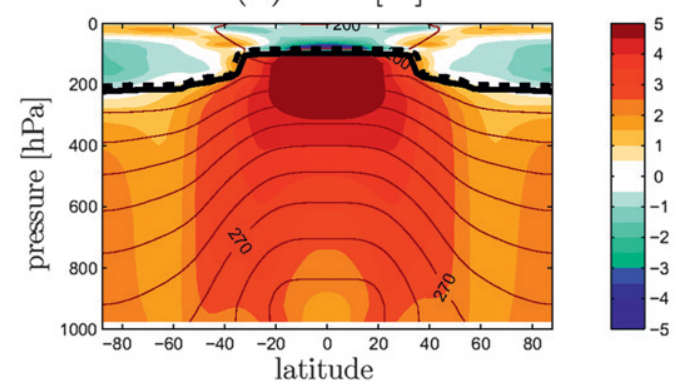

(e) $\Delta u\left[\mathrm{~m} \mathrm{~s}^{-1}\right]$

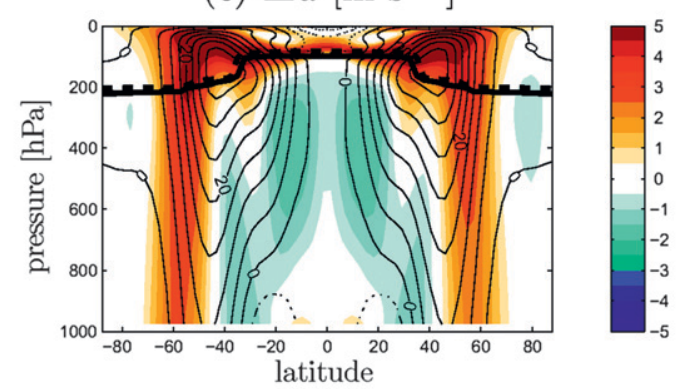

(f) $\Delta \Psi\left[10^{9} \mathrm{~kg} \mathrm{~s}^{-1}\right]$

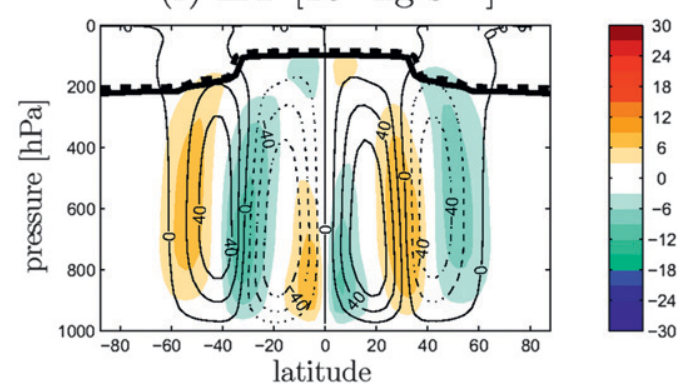

FIG. 4. As in Fig. 2, but for (a)-(c) the Phi35 $5_{\mathrm{LT}}$ integration, in which there is no lapse-rate perturbation; and (d)-(f) the Phi35 $5_{\mathrm{UT}}$ integration, in which the lapse-rate perturbation is twice that of the standard Phi35 integration.

perturbation results in the peak warming being located in the lower troposphere rather than the upper troposphere. However, in terms of the shifts of the jet and the HC edge, there appears to be little difference between the LT integrations and the standard ones. The LT results show a slight negative offset from their standard integration counterparts, except for a slight positive offset for the jet shift in the Phi5 $5_{\mathrm{LT}}$ and Phi15 $5_{\mathrm{LT}}$ cases.

Figures $4 \mathrm{a}-\mathrm{c}$ show the response of the Phi35 $5_{\mathrm{LT}}$ integration in more detail. Comparing the temperature response (Fig. 4a) with that of Phi35 (Fig. 2d), we see much less warming in the tropical upper troposphere and enhanced warming in the lower troposphere. Phi35 does show some westward acceleration in the tropical upper troposphere that is not apparent in Phi35 $5_{\mathrm{LT}}$ (cf. Fig. 2e and Fig. 4b), but aside from that, the circulation responses are nearly indistinguishable. When we compare the other LT integrations to the standard integrations, the differences are all minor. The most noticeable differences are in the Phi5 $5_{\mathrm{LT}}$ integration (not shown): at the equator, there is no westward acceleration at upper levels, no deepening of the HC, and no vertical deceleration near the surface. (compare this with Figs. 2b,c.) As noted above, our Phi5 integration produces much greater westward acceleration at the equator than in comprehensive model simulations of El Niño, so our results suggest that convection plays an important role in the equatorial circulation response. As for the Phi35-20 ${ }_{\mathrm{LT}}$ integration (not shown), the zonal wind response is slightly more barotropic than that of Phi35-20 (Fig. 2h). 
(a)

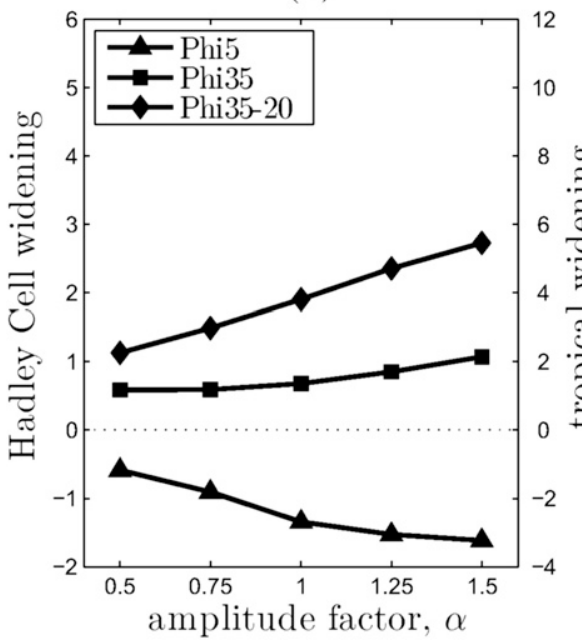

(b)

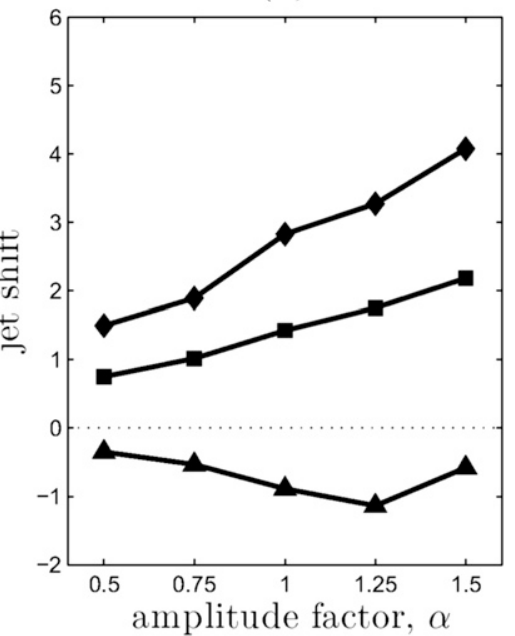

FIG. 5. Changes in circulation metrics as functions of relative forcing amplitude $\alpha$. The circulation metrics are defined in the caption of Fig. 3 and in the text.

We have also tested the effect of imposing a larger decrease of the lapse rate. These integrations are given the "UT" label and are plotted in green in Fig. 3. These integrations show a slight positive offset from the standard integrations. So for the global warming-like (large $\left.\phi_{w}\right)$ cases, decreasing the lapse rate does result in enhanced HC expansion, but this effect is small compared to the effect of changing $\phi_{w}$. Overall, the circulation responses of the UT integrations are qualitatively similar to those of the standard integrations, but there are notable quantitative differences. For example, in the Phi35 Ut case (Fig. 4, right column), features that were barely noticeable in the Phi35 integration become more pronounced, like the local minimum in warming in the tropical lower troposphere (Fig. 4d), the westward acceleration around the equator (Fig. 4e), and the dipole streamfunction anomalies near the surface and near the tropopause at the equator (Fig. 4f). These results, together with those of the LT integrations, suggest that the circulation responses are sensitive more to the horizontal structure of the thermal forcing than to its vertical structure. It is possible, however, that thermal forcings with more complicated vertical structure might produce different results.

We have also performed a set of integrations in which we sweep the relative amplitude of the thermal forcing by varying the factor $\alpha$, defined in (1). One might expect that the responses are linear in $\alpha$, in which case a doubling of the forcing amplitude should double the amount of HC expansion and jet shift. The results shown in Fig. 5 are approximately linear, except for the Phi5 integrations at high $\alpha$, which even show some nonmonotonicity (Fig. 5b, triangles). The responses do not exhibit any jump discontinuity like that shown in Wang et al. (2012), even though the amplitudes of our thermal forcings are comparable. The Phi5 and Phi35 integrations show slight nonlinearity at low $\alpha$, but the circulation responses are very weak in these cases, so much longer integrations would be required to confirm a statistically robust nonlinearity. It is also clear that the Phi35-20 response is well-separated from that of Phi35: even if we reduce the amplitude of the Phi35-20 forcing by half $(\alpha=0.5)$, the response is still greater than the Phi35 response at its default amplitude.

The relatively large circulation response of Phi35-20, detailed above, suggests that there might be a linearly additive relationship between the responses to wide and narrow thermal forcings. To test this more rigorously, we have performed Phi35-20 ${ }_{\mathrm{LT}}$ and Phi20 $0_{\mathrm{LT}}$ integrations with their forcing amplitudes chosen so that their sum matches the exact amplitude of the Phi35 $5_{\mathrm{LT}}$ forcing. This requires that we set $\alpha=15 / 35$ for the Phi35-20 ${ }_{\mathrm{LT}}$ forcing and $\alpha=20 / 35$ for the Phi20 forcing [see (1) and (3)]. In this case, we find that Phi35-20 $\mathrm{LT}_{\mathrm{LT}}$ produces $0.63^{\circ} \pm 0.05^{\circ} \mathrm{HC}$ expansion, compared to $0.54^{\circ} \pm 0.06^{\circ}$ for Phi35 $\mathrm{LT}$ and $-0.02^{\circ} \pm$ $0.02^{\circ}$ for Phi20 ${ }_{\text {LT }}$. (Negative values indicate $\mathrm{HC}$ contraction.) So the Phi35-20 $\mathrm{LT}$ response is larger than the difference of the Phi35 $5_{\mathrm{LT}}$ and Phi20 $0_{\mathrm{LT}}$ responses, but this nonlinearity is not statistically significant.

\section{A diffusive model of the circulation response}

\section{a. Approach}

The key result from our GCM experiments is that the transition from $\mathrm{HC}$ contraction to $\mathrm{HC}$ expansion is 
determined primarily by the meridional width of the thermal forcing. We now seek a simplified explanation of this behavior. To begin, we remind ourselves that the $\mathrm{HC}$ edge coincides with a downward maximum of the zonal mean vertical velocity, $\bar{\omega}$. So if we wish to determine how the $\mathrm{HC}$ edge shifts in response to a particular thermal forcing, then we need to relate $\bar{\omega}$ to the net diabatic heating $\overline{\mathbb{Q}}$. Fortunately, these quantities are directly related through the temperature equation, but the temperature equation includes additional contributions, most important of which is the divergence of the meridional eddy heat flux $\overline{v^{\prime} \theta^{\prime}}$.

Thus the challenge is finding a way to represent the circulation that makes the problem tractable. To this end, we choose to parameterize the total circulation as diffusive, following an approach similar to that of Frierson et al. (2007a) and Kang et al. (2009). This parameterization accounts for transport due to both eddies and the mean flow by assuming that they together act to diffuse heat meridionally. Such an approach greatly simplifies the system, but in the process, it blurs the distinction between eddies and the mean flow. This makes it more appropriate that we work in terms of the transformed Eulerian mean (Edmon et al. 1980), which combines the Eulerian vertical velocity and eddy heat flux divergence into a single quantity representing the total heat transport. This quantity is called the residual vertical velocity $\bar{\omega}^{*}$ and it is defined as

$$
\bar{\omega}^{*} \equiv \bar{\omega}+\frac{1}{a \cos \phi} \frac{\partial}{\partial \phi}\left(\frac{\overline{v^{\prime} \theta^{\prime}} \cos \phi}{\bar{\theta}_{p}}\right),
$$

where $\bar{\theta}_{p}$ is the vertical stratification in pressure coordinates, $\phi$ is latitude, and $a$ is Earth's radius.

This raises a pivotal question: how do we locate the $\mathrm{HC}$ edge in the TEM system? The TEM meridional circulation consists of just one cell extending from the equator to the pole (Edmon et al. 1980), in contrast to the three-cell structure of the Eulerian mean circulation. However, we can still identify the HC from the TEM circulation. This is because, in the upper troposphere, eddy heat fluxes are small enough that there is a close correspondence between $\bar{\omega}^{*}$ and $\bar{\omega}$. As seen in Edmon et al. (1980), Fig. 6a, or Held and Schneider (1999), Fig. $3 \mathrm{a}$, the upper half of the HC is clearly evident in the upper tropospheric portion of the TEM circulation, where the Eulerian mean flow dominates.

We have found that the $\mathrm{HC}$ edge can be accurately identified as the latitude where there is a downward maximum of $\bar{\omega}^{*}$ when averaged over $200-500 \mathrm{hPa}$; we call this quantity $\bar{\omega}_{\max }^{*}$. By vertically averaging over the upper troposphere, we ensure that the maximum is

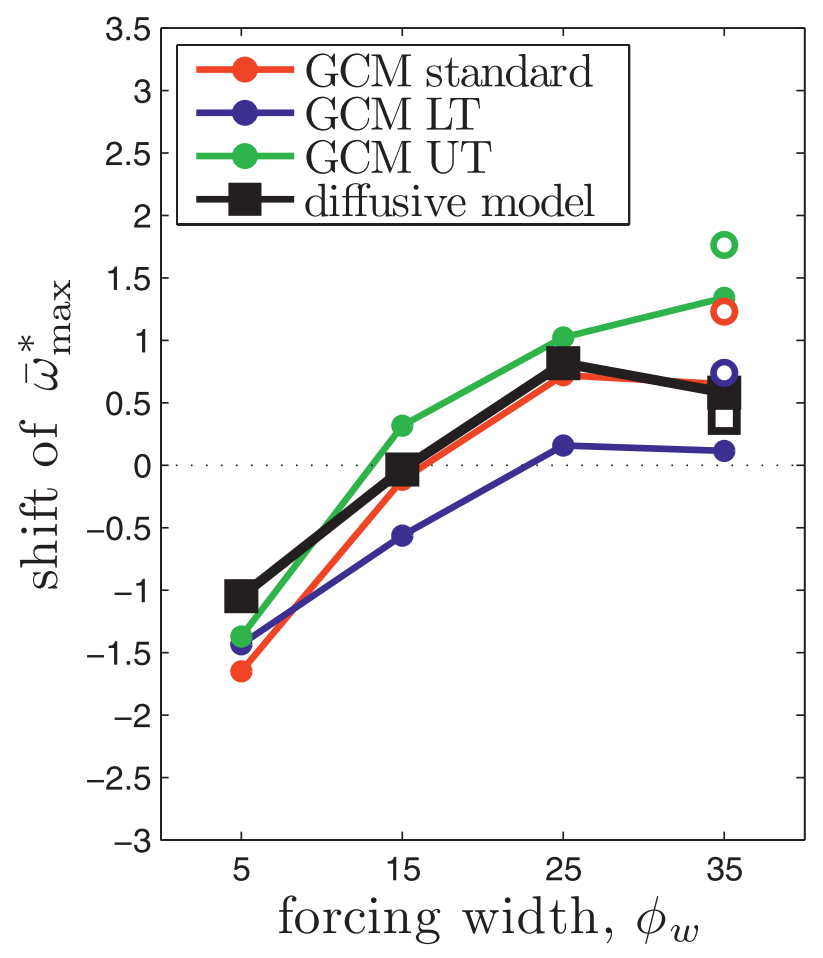

FIG. 6. Shift of the downward maximum of residual vertical velocity in the upper troposphere $\bar{\omega}_{\max }^{*}$ as a function of the meridional width of the thermal forcing $\phi_{w}$. Red circles refer to standard GCM integrations, blue circles refer to LT integrations of the GCM, and green circles refer to UT integrations of the GCM. Black squares show results from the diffusive model described in Sec. 3. Empty markers indicate results from the Phi35-20 cases.

robustly located. Most importantly for our purposes, this definition accurately captures changes in $\mathrm{HC}$ width due to thermal forcings. Figure 6, circles, shows the shift of $\bar{\omega}_{\max }^{*}$ from the GCM experiments of section 2. Comparing Fig. 6 with Fig. 3, one sees that the $\bar{\omega}_{\max }^{*}$ metric and the conventional $\Psi_{500}$ metric agree well with each other; the modest differences that do arise are not substantial enough to affect our key conclusions.

Defining the $\mathrm{HC}$ edge in terms of $\bar{\omega}^{*}$ is a key step because we can obtain a very simple relation between the change in $\bar{\omega}^{*}$ and the anomalous diabatic heating. This, combined with our diffusive parameterization of the circulation, allows us to solve for the change in residual vertical velocity, and thus the shift of the HC edge (i.e., $\bar{\omega}_{\max }^{*}$ ). Not surprisingly, this diffusive model has important limitations, which we address below. Nonetheless, the model provides a very simple way of understanding the transition from $\mathrm{HC}$ contraction to $\mathrm{HC}$ expansion.

\section{b. Mathematical formulation}

Having outlined our approach, we now provide the formal details. Our domain is taken to be the arc 
spanning $0^{\circ}-90^{\circ}$ latitude, representing a layer averaged zonally and vertically over the upper troposphere of one hemisphere. (We assume hemispheric symmetry.) In the TEM system, the temperature equation takes the form

$$
\frac{\partial \bar{\theta}}{\partial t}+\bar{\theta}_{p} \bar{\omega}^{*}=\overline{\mathbb{Q}},
$$

where $\bar{\theta}$ is the zonal mean potential temperature, and $t$ is time. We hereafter refer to $\overline{\mathbb{Q}}$ as the "diabatic tendency," and this term can be positive (diabatic heating) or negative (diabatic cooling). In contrast to the system considered by Held and Hou (1980), (5) neglects horizontal advection by the mean flow, but implicitly includes eddy heat flux divergence.

We assume steady-state conditions and parameterize the diabatic tendency as Newtonian cooling, so (5) becomes

$$
\bar{\theta}_{p} \bar{\omega}^{*}=-\frac{\bar{\theta}-\bar{\theta}_{\mathrm{eq}}}{\tau},
$$

where $\bar{\theta}_{\text {eq }}$ is the equilibrium potential temperature, and $\tau$ is the relaxation time scale. This means that temperature deviations from thermal equilibrium must be balanced by vertical advection. If we were to neglect eddy heat fluxes, (6) would reduce to a form equivalent to that obtained under the weak temperature gradient (WTG) approximation (e.g., Held and Hoskins 1985; Sobel et al. 2001), as well as other linear formulations of the tropical circulation (e.g., Schneider and Lindzen 1976; Gill 1980; Wang and $\mathrm{Li}$ 1993). We consider this eddy-neglecting limit further below.

Thus our system has two unknowns: $\bar{\omega}^{*}$ and $\bar{\theta}$. To close the system, we parameterize the TEM circulation by assuming that vertical advection acts to diffuse potential temperature meridionally. Specifically,

$$
\bar{\theta}_{p} \bar{\omega}^{*}=-\frac{k}{a^{2} \cos \phi} \frac{\partial}{\partial \phi}\left(\cos \phi \frac{\partial \bar{\theta}}{\partial \phi}\right),
$$

where $k$ is the diffusivity, taken to be spatially uniform. We eliminate $\bar{\omega}^{*}$ by (6) and (7), obtaining

$$
\bar{Q}-\frac{\bar{\theta}}{\tau}=-\frac{k}{a^{2} \cos \phi} \frac{\partial}{\partial \phi}\left(\cos \phi \frac{\partial \bar{\theta}}{\partial \phi}\right),
$$

where $\bar{Q}$ is the diabatic source term, defined as $\bar{Q}=\bar{\theta}_{\mathrm{eq}} / \tau$. This means that meridional diffusion acts to balance the diabatic tendency. This is analogous to the formulations of Frierson et al. (2007a) and Kang et al. (2009), in which the meridional diffusion of moist static energy acts to balance the net radiative heating.
We now perturb the system with a thermal forcing $\tilde{Q}$. This in turn produces perturbations of temperature $\tilde{\theta}$ and residual vertical velocity $\tilde{\omega}^{*}$. For simplicity, we assume that the diffusivity and stratification remain fixed. We separate the perturbations from their associated background values, so that

$$
\begin{aligned}
\langle\bar{Q}\rangle & =\bar{Q}+\tilde{Q}, \\
\langle\bar{\theta}\rangle & =\bar{\theta}+\tilde{\theta}, \quad \text { and } \\
\left\langle\bar{\omega}^{*}\right\rangle & =\bar{\omega}^{*}+\tilde{\omega}^{*},
\end{aligned}
$$

where angle brackets denote final values after the perturbation. Placing these into (6) and (8), we can subtract the background state and obtain equations for just the perturbation fields:

$$
\begin{aligned}
\tilde{Q}-\frac{\tilde{\theta}}{\tau} & =-\frac{k}{a^{2} \cos \phi} \frac{\partial}{\partial \phi}\left(\cos \phi \frac{\partial \tilde{\theta}}{\partial \phi}\right) \quad \text { and } \\
\tilde{\omega}^{*} & =\frac{1}{\bar{\theta}_{p}}\left(\tilde{Q}-\frac{\tilde{\theta}}{\tau}\right) .
\end{aligned}
$$

The quantity $\tilde{Q}-\tilde{\theta} / \tau$ represents the anomalous diabatic tendency. Thus in the case of stable stratification $\left(\bar{\theta}_{p}<0\right)$, anomalous diabatic heating $(\tilde{Q}-\tilde{\theta} / \tau>0)$ is balanced by anomalous TEM ascent $\left(\tilde{\omega}^{*}<0\right)$. In $(12)$ is a one-dimensional boundary value problem in $\tilde{\theta}$. The boundary conditions are taken to be $\partial \tilde{\theta} / \partial \phi=0$ at the equator (by hemispheric symmetry) and $\partial \tilde{\theta} / \partial \phi=0$ at the pole (to maintain thermal wind balance with zero zonal wind). Once we solve (12) for $\tilde{\theta}$, then we can solve (13) for $\tilde{\omega}^{*}$.

Since we are primarily interested in the shift of the $\mathrm{HC}$ edge, we use this diffusive model to compute only perturbation fields. (This is not a model for the mean Hadley circulation.) The background state is obtained from output of our GCM control integration; this output is zonally and vertically averaged over $200-500 \mathrm{hPa}$, and values from both hemispheres are combined to double the sample size. We apply the same averaging scheme when comparing the GCM responses to the results of the diffusive model (see below). The parameters of the diffusive model are chosen as follows: We let $\bar{\theta}_{p}=-4 \times 10^{-4} \mathrm{~K} \mathrm{~Pa}^{-1}$, which matches the vertical stratification in the upper troposphere of the GCM control integration. Second, we find that the temperature response of the diffusive model adequately matches that of the GCM if we let $k=1 \times 10^{6} \mathrm{~m}^{2} \mathrm{~s}^{-1}$ and $\tau=35$ days. This value for $k$ is of the same order as that used in Frierson et al. (2007a) and Kang et al. (2009), and the value for $\tau$ is comparable to other estimates of the thermal 
(a) Phi5

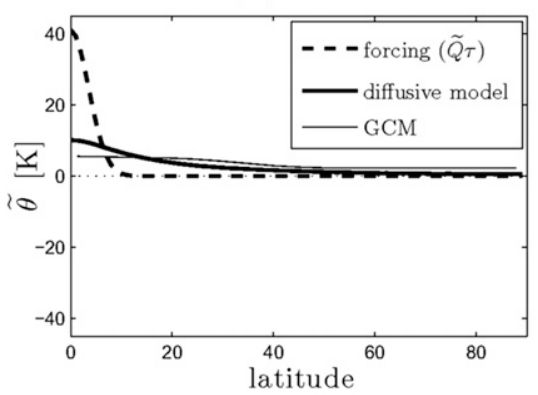

(d)

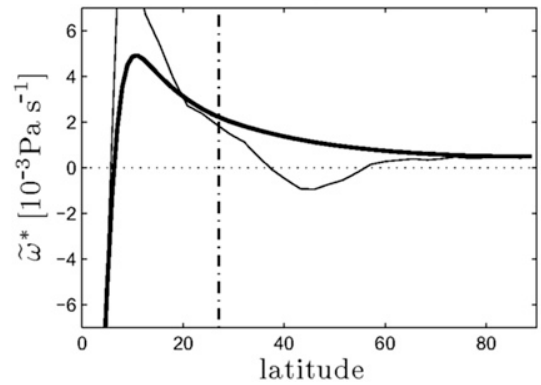

(b) Phi15

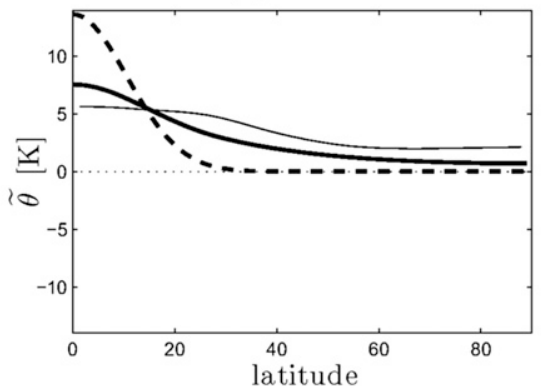

(e)

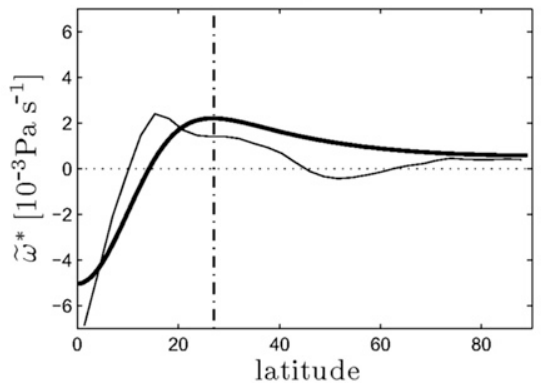

(c) Phi25

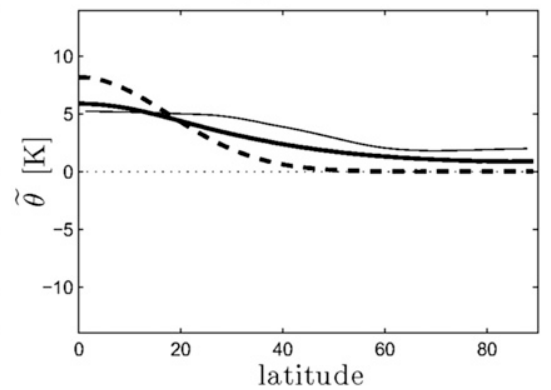

(f)

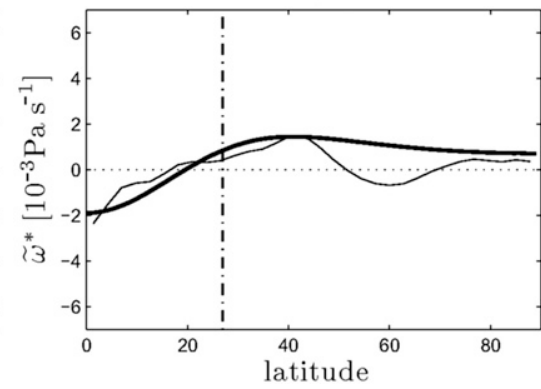

FIG. 7. (a)-(f) Results from the diffusive model described in section 3 for the forcings indicated at the top of each column. Thick solid lines show output from the diffusive model. Thin black lines show output from the standard GCM integrations, shown for comparison. Thick dashed lines show the imposed thermal forcings in units of temperature (i.e., $\tilde{Q} \tau)$. The vertical dot-dashed lines in the bottom panels indicate the latitude of the HC edge (i.e., $\bar{\omega}_{\max }^{*}$ ) from the control integration. Note, for clarity the vertical scale of panel (a) is different from the other panels.

equilibrium time scale in the troposphere (Held and Suarez 1994; Robinson 2002). The thermal forcings ( $\tilde{Q})$ used in the diffusive model are equal to the thermal forcings used in the GCM integrations, vertically averaged over 100-1000 hPa. We average the thermal forcings over the whole troposphere (rather than just the upper troposphere) to account for the fact that convection spreads the thermal forcing vertically.

\section{c. Results}

Figure 7 shows numerical solutions of the diffusive model. The dashed curves in the top row show the thermal forcings $\tilde{Q}$, multiplied by $\tau$. These represent what the temperature responses would be if there were no changes in the circulation. The $e$-folding widths of the thermal forcings range from $5^{\circ}$ (Phi5) in the leftmost column to $25^{\circ}$ (Phi25) on the right. The thick solid curves in the top panels show the calculated temperature responses. By construction, these show a diffusive character: the temperature responses are flattened compared to $\tilde{Q} \tau$. Thus, there is a transition from anomalous diabatic heating $(\tilde{Q} \tau>\tilde{\theta})$ in the region of peak thermal forcing to anomalous diabatic cooling $(\tilde{Q} \tau<\tilde{\theta})$ elsewhere.

The bottom panels of Fig. 7 show the responses of the residual vertical velocity. As follows directly from
(13), there is anomalous ascent in regions of anomalous diabatic heating (i.e., $\tilde{\omega}^{*}<0$ for $\tilde{Q} \tau>\tilde{\theta}$ ) and anomalous descent in regions of anomalous diabatic cooling (i.e., $\tilde{\omega}^{*}>0$ for $\left.\tilde{Q} \tau<\tilde{\theta}\right)$. Thus, there is anomalous descent on the poleward flank of the thermal forcing. The vertical dot-dashed lines in the bottom panels mark the edge of the HC (i.e., $\bar{\omega}_{\max }^{*}$ ) calculated from the GCM control integration. The results show that for the Phi5 case (Fig. $7 \mathrm{~d}$ ), there is a descending anomaly whose maximum is on the equatorward side of the $\mathrm{HC}$ edge, producing contraction of the HC. As the thermal forcing is widened, the peak of this descending anomaly moves to the poleward side of the $\mathrm{HC}$ edge (Figs. 7e,f), resulting in expansion of the HC. Thus, our simple diffusive model qualitatively reproduces the transition from $\mathrm{HC}$ contraction to HC expansion.

For comparison purposes, the thin black lines in Fig. 7 show the same fields obtained from the standard GCM integrations. For the temperature responses (top row), the main discrepancy is that the GCM responses have less meridional gradient in the low- to midlatitudes when compared to the diffusive model. Better agreement may be achieved by spatially varying the diffusivity, but this would not affect any of the key conclusions drawn from the model. As for the residual vertical 
velocity (bottom row), the main discrepancy is that the GCM responses show ascending anomalies in the midlatitudes, which are completely missing in the diffusive model. Calculating heat budget terms from the GCM (not shown), we find that these ascending anomalies are primarily associated with anomalies of the vertical eddy heat flux $\left(\overline{\omega^{\prime} \theta^{\prime}}\right)$, which is neglected in the TEM approximation. Edmon et al. (1980) have also noted the importance of vertical eddy heat fluxes in the midlatitudes. This discrepancy, however, occurs far enough poleward of the $\mathrm{HC}$ edge that it does not contribute significantly to the shift of the HC edge, except possibly in the Phi5 case.

Next, as a more quantitative test, we add $\tilde{\omega}^{*}$ from the diffusive model to the climatological $\bar{\omega}^{*}$ of the GCM and calculate the resulting shift of the $\mathrm{HC}$ edge (i.e., $\bar{\omega}_{\max }^{*}$ ). This is plotted as the black squares in Fig. 6. The diffusive model shows close quantitative correspondence with the output of the GCM (red circles), both in terms of the amplitude of $\mathrm{HC}$ expansion, as well as the transition from $\mathrm{HC}$ contraction to $\mathrm{HC}$ expansion. One point of disagreement is that the diffusive model produces about one degree less $\mathrm{HC}$ contraction than the GCM for the Phi5 integration. This may be due to the fact that, compared to the diffusive model, the GCM produces more descent just equatorward of the $\mathrm{HC}$ edge and more ascent just poleward of the HC edge (Fig. 7d). As noted above, the latter anomaly is associated with vertical eddy heat fluxes, which the diffusive model does not capture (Fig. 7d).

A bigger discrepancy in Fig. 6 is that the diffusive model does not reproduce the much-enhanced $\mathrm{HC}$ expansion seen in the Phi35-20 case. Instead, the diffusive model produces slightly less HC expansion for Phi35-20 (empty black square) than it does for Phi35. Figure 8 shows the output of the diffusive model for the Phi35-20 forcing. In this case, the model produces a broad ascending anomaly that peaks slightly equatorward of the HC edge (Fig. 8b, thick line). In contrast, the GCM shows a sharp, spatially confined ascending anomaly on the equatorward flank of the $\mathrm{HC}$ edge, and a similarly sharp descending anomaly on its poleward flank (Fig. 8b, thin solid line). Further examination of GCM output reveals that this dipole anomaly coincides with a similarly pronounced dipole of anomalous eddy momentum flux convergence/divergence (not shown).

Thus, this discrepancy appears to be due to our model's inability to capture the effects of eddy momentum fluxes, which cannot be modeled as a simple diffusive process. Eddy momentum fluxes might also be partly responsible for driving the anomalous vertical eddy heat fluxes associated with other model discrepancies noted above. It is worth noting that for the thermal forcings in Fig. 7, the peaks of the thermal forcings (a) Phi35-20

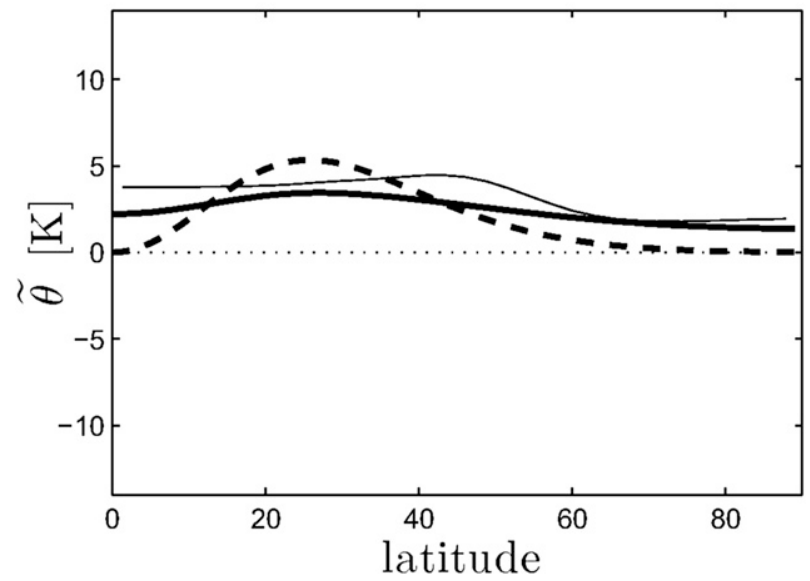

(b)

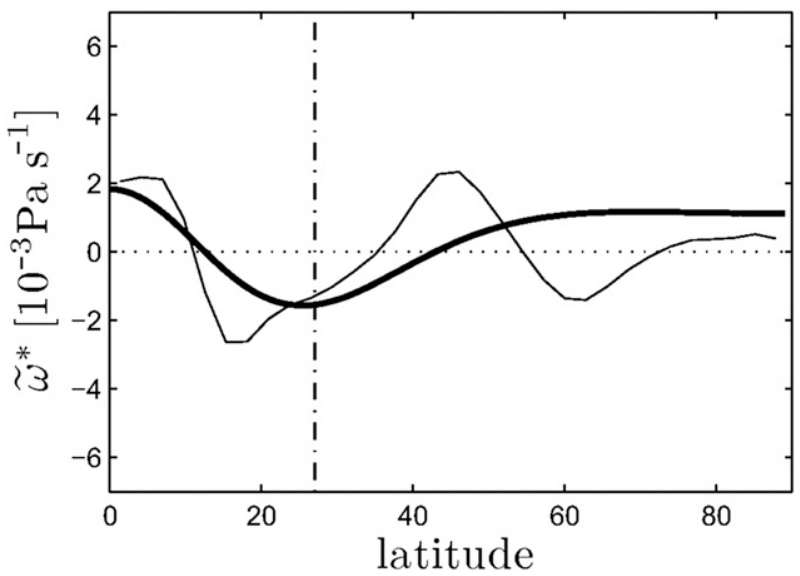

FIG. 8. As in Fig. 7, but for the Phi35-20 case.

(and thus the peaks of the ascending anomalies) are situated at the equator, where the eddy momentum flux is negligible. Meanwhile, in the Phi35-20 case, the entire thermal forcing is situated in a region where normally there is substantial flux of eddy momentum. This may be a crucial aspect of the Phi35-20 forcing that leads to the discrepancy between the diffusive model and the GCM.

Earlier studies have applied the thermal wind balance principle to relate shifts of the midlatitude jet to changes in the meridional temperature gradient (Seager et al. 2003; Lorenz and DeWeaver 2007; Allen et al. 2012). It is tempting to use our diffusive model to calculate the jet shift from the temperature response, but the model is not suitable for this purpose. This is because the diffusive model produces a temperature response whose meridional gradient lacks important structure. For example, in the Phi15 case (Fig. 7b), the diffusive model's temperature response has its steepest gradient between $10^{\circ}$ and $30^{\circ}$ latitude, whereas the GCM response is nearly 
flat through this region and has its steepest gradient farther poleward, between $35^{\circ}$ and $45^{\circ}$ latitude. This difference is substantial enough that the diffusive model would produce shifts of the midlatitude jet that are highly inaccurate. This shortcoming of the diffusive model is not surprising, since eddy momentum fluxes are believed to play an important role in shifting the midlatitude jet (Seager et al. 2003; Wu et al. 2012; Chen et al. 2013), and our model, as noted above, is incapable of properly capturing them.

As an additional test, we have calculated the shift of the $\mathrm{HC}$ edge assuming there is no contribution from the meridional eddy heat flux. Such an assumption, as noted above, is common to linear models of the tropical circulation, and it means that there is no need to distinguish between the residual vertical velocity and the Eulerian vertical velocity (i.e., $\bar{\omega}^{*}=\bar{\omega}$ ). If we also assume the same scalings as used for the TEM equations, then the change in Eulerian vertical velocity $\tilde{\omega}$ is obtained directly from (13).

In this eddy-neglecting limit, we have used our diffusive model to calculate $\tilde{\omega}$ for each thermal forcing. Adding this change to the climatological $\bar{\omega}$ from the GCM control integration, we have also calculated the shift of the maximum of $\bar{\omega}$, which coincides with the HC edge. In this case (not shown), we obtain a transition from $\mathrm{HC}$ contraction to $\mathrm{HC}$ expansion at approximately the same value of $\phi_{w}$, but the actual magnitude of HC expansion is about an order of magnitude lower than that shown in Figs. 3a and 6. Therefore, to obtain a reasonable amplitude of HC expansion, we cannot assume that eddy heat fluxes are unchanged; changes in eddy heat fluxes appear to be a key contribution. This does not clarify whether the circulation response is actually driven by eddy heat fluxes, as suggested by Butler et al. (2011), rather than eddy momentum fluxes, as argued by others (Seager et al. 2003; Wu et al. 2012; Chen et al. 2013).

In any case, our diffusive model does demonstrate that the circulation response can be understood largely in terms of thermally driven processes. That is, a positive thermal forcing produces anomalous TEM descent on its poleward flank. If this anomalous descent is located equatorward (poleward) of the $\mathrm{HC}$ edge, then the $\mathrm{HC}$ contracts (expands).

\section{Discussion}

\section{a. Changes in baroclinicity}

Earlier studies have examined the degree to which $\mathrm{HC}$ width obeys the scalings suggested by baroclinic instability theory (e.g., Held 2000; Walker and Schneider
2006; Frierson et al. 2007b, Lu et al. 2008). Using the baroclinic criticality formulation of Phillips (1954), Lu et al. (2008) showed that a decrease in criticality is associated with a poleward shift of the HC edge. Phillips' criticality depends on both bulk vertical shear and bulk static stability, but Lu et al. (2008) showed results suggesting that increased static stability is the dominant contributor to HC expansion in coupled GCM. Lu et al. (2010) arrived at a similar conclusion when varying the SST forcing in an atmosphere-only GCM. These findings are seemingly at odds with our LT integrations, which produce significant $\mathrm{HC}$ expansion even when tropical static stability decreases (e.g., Fig. 4). We must emphasize, however, that the relevant changes in baroclinicity depend on static stability changes in the subtropics (i.e., on the equatorward flank of the jet), not the tropics.

Thus, to properly compare with earlier findings, we have calculated from our GCM output the change in Phillips' criticality using the same formulations as in $\mathrm{Lu}$ et al. (2008). Specifically, we compute the difference in criticality $\delta C$ between each of our forced integrations and our control integration,

$$
\delta C=\delta\left[\frac{f^{2}\left(u_{500}-u_{850}\right)}{\beta g H\left(\theta_{500}-\theta_{850}\right) / \Theta_{0}}\right],
$$

where $u$ is the zonal wind, $g$ is the gravitational acceleration, $f$ is the Coriolis parameter, $\beta$ is the meridional gradient of the Coriolis parameter, $H$ is the height scale, $\Theta_{0}$ is a reference temperature, and the 500 and 850 subscripts indicate the pressure levels, in hectopascals, where $u$ and $\theta$ are evaluated. This expression is then expanded into contributions due to static stability,

$$
\delta C_{\mathrm{st}} \approx-\frac{f^{2}\left(u_{500}-u_{850}\right)_{\mathrm{ctl}} \delta\left(\theta_{500}-\theta_{850}\right)}{\beta g H\left(\theta_{500}-\theta_{850}\right)_{\mathrm{ctl}}^{2} / \Theta_{0}},
$$

and vertical shear,

$$
\delta C_{\mathrm{sh}}=\frac{f^{2} \delta\left(u_{500}-u_{850}\right)}{\beta g H\left(\theta_{500}-\theta_{850}\right)_{\mathrm{ctl}} / \Theta_{0}},
$$

where the ctl subscript indicates quantities calculated from the control integration. These expressions indicate that the criticality can be reduced either by increasing static stability or by decreasing vertical shear. To compute these quantities from GCM output, we first meridionally average the zonal-mean wind and potential temperature fields over $21^{\circ}-46^{\circ}$ latitude (which is the $25^{\circ}$ band immediately equatorward of the midlatitude jet of the control integration, following $\mathrm{Lu}$ et al. 2008). Then we apply (14)-(16) with $H=5 \mathrm{~km}, \Theta_{0}=300 \mathrm{~K}$, and $f$ 
(a)

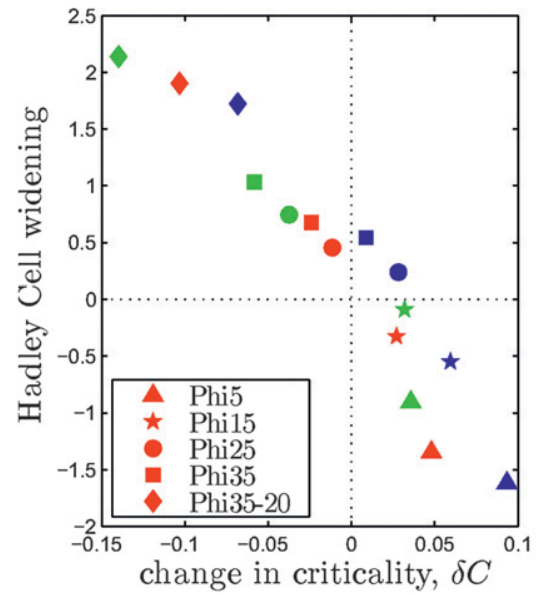

(b)

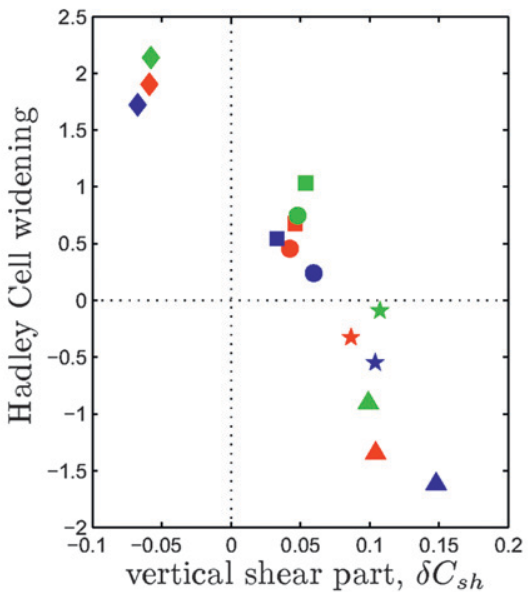

(c)

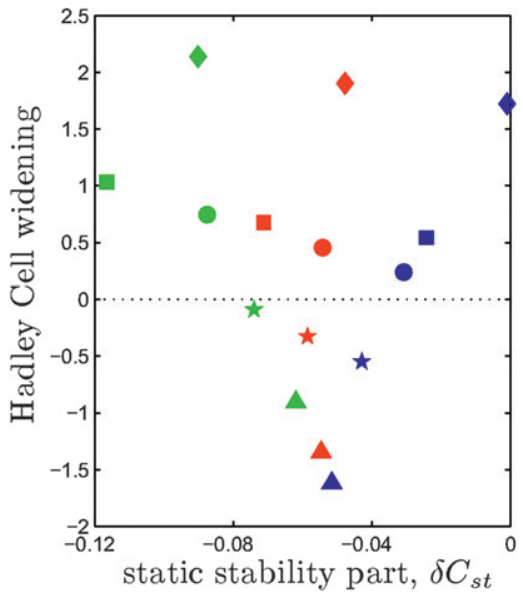

FIG. 9. Relationships between HC widening and (a) change in total Phillips' criticality $\delta C$, (b) change in criticality due to bulk vertical shear $\delta C_{\mathrm{sh}}$, and (c) change in criticality due to bulk static stability $\delta C_{\mathrm{st}}$. Red markers indicate standard integrations, blue markers indicate LT integrations, and green markers indicate UT integrations. Results from the Northern and Southern Hemispheres have been averaged together. Note that negative values for $\delta C_{\mathrm{sh}}$ indicate decreases in vertical shear, and negative values for $\delta C_{\mathrm{st}}$ indicate increases in static stability.

and $\beta$ computed at $33.5^{\circ}$ (the midpoint of the latitude band).

We present the results of these calculations in Fig. 9. Specifically, Fig. 9a shows the change in $\mathrm{HC}$ width versus the change in total criticality, $\delta C$. This shows that, in agreement with earlier studies, decreases (increases) in criticality are generally associated with $\mathrm{HC}$ expansion (contraction). Figure $9 \mathrm{~b}$ plots $\mathrm{HC}$ widening versus $\delta C_{\mathrm{sh}}$. This exhibits a pattern similar to that of Fig. 9a, although the data are shifted farther from the origin: several integrations show increases in $\delta C_{\mathrm{sh}}$ associated with $\mathrm{HC}$ expansion. Figure $9 \mathrm{c}$ shows $\mathrm{HC}$ widening versus $\delta C_{\mathrm{st}}$, and the results here are widely scattered, with the LT integrations (blue markers) even showing a positive correlation between $\delta C_{\mathrm{st}}$ and $\mathrm{HC}$ width.

Thus our results disagree with those of Lu et al. (2008, 2010): changes in vertical shear-not static stabilityappear to be the dominant contributor to HC expansion in our model. This contrast may be due to the fact that our model is dry, and thus changes in static stability are not constrained in the same way as in moist models. Another possible explanation is that $\mathrm{Lu}$ et al. (2008, 2010) consider a more narrow range of forcings than we do, and that a different selection of forcings in comprehensive models might produce $\mathrm{HC}$ expansion with a more significant vertical shear contribution.

\section{b. Jet position versus Hadley Cell edge}

Earlier studies (e.g., Fu et al. 2006; Seidel et al. 2008; Fu and Lin 2011; Davis and Rosenlof 2012) have used the position of the jet to examine the widening trend of the tropics. Our results suggest that using a metric based on jet latitude rather than $\mathrm{HC}$ edge can give a different impression of how the width of the tropical belt is changing. Figure 3 shows that the shift of the HC edge and the shift of the jet can be quite different for the same thermal forcing. If one is more interested in the location of the dry zones, which is closely related to the location of the $\mathrm{HC}$ edge, then relying on a jet latitude metric would be somewhat misleading.

This difference between jet latitude and HC edge may relate to the fact that the subtropical jet and the midlatitude eddy-driven jet can separate from each other. The precise drivers of this jet separation remain unclear. Lu et al. (2008) took an initial step by showing that in coupled model simulations of global warming, the poleward shift of the SH midlatitude jet is about twice the shift of the $\mathrm{HC}$ edge. This result agrees with our global warming-like integrations (Fig. 3 for large $\phi_{w}$ ) but not with our El Niño-like integrations (Fig. 3 for small $\left.\phi_{w}\right)$. To further complicate matters, Kang and Polvani (2011) showed that in coupled models, there is no correlation between $\mathrm{HC}$ edge and jet latitude during winter in SH and during all seasons in NH. Thus, many questions remain in this area.

\section{c. Warming in the upper versus lower troposphere}

The results of Figs. 3 and 4 suggest that our lapse-rate perturbation has relatively little effect on the circulation response. This does not mean that warming in the upper troposphere is less important than warming in the lower troposphere. Note that for the Phi35 $5_{\mathrm{LT}}$ integration (Fig. 4), 
even though the thermal forcing is confined to the lower troposphere, there is still significant warming in the upper troposphere. We have also performed an integration in which the thermal forcing is more strictly confined to the upper troposphere between $-35^{\circ}$ and $35^{\circ}$ latitude (not shown). We accomplish this by increasing the amplitude of the lapse-rate perturbation and reducing the amplitude of the thermal forcing in the lower troposphere. The associated temperature response is comparable to the upper tropospheric response of Phi35 (Fig. 2d), but there is much less warming in the lower troposphere. Despite this change in the vertical structure of the warming, the resulting $\mathrm{HC}$ expansion and poleward shift of the jet is nearly equal to that of Phi35. This gives further support to our earlier finding: there is relatively little sensitivity to the change in the lapse rate, and there is much greater sensitivity to the meridional structure of the thermal forcing.

There is, however, a caveat to this claim: a narrow thermal forcing confined to the tropical upper troposphere produces a response that is not completely $\mathrm{El}$ Niño-like. In this case, the HC contracts slightly, but the jets shift poleward. Thus, warming in the tropical lower troposphere appears to be essential for producing an El Niño-like circulation response. The reasons for this sensitivity are unclear, but we would argue that such a thermal forcing is highly unrealistic. Specifically, warming in the tropical upper troposphere would typically require some warming in the tropical lower troposphere as well, especially in the case of El Niño, where there is substantial warming at the surface.

In the context of global warming, however, our results suggest that the lapse-rate feedback is not as consequential for the tropospheric circulation as earlier studies hypothesize (Butler et al. 2010, 2011; Wang et al. 2012). We obtain much the same circulation response whether peak warming occurs in the upper troposphere or the lower troposphere.

\section{d. Implications for recent observations}

While the results of our Phi35 integration resemble the global warming response of comprehensive models, it is not certain that this accurately represents the trends observed over recent decades. Satellite observing systems have been used to study the trend in vertically averaged tropospheric temperatures, and these suggest that recent warming has been maximum over the Arctic and in the midlatitudes (Santer et al. 2003; Fu et al. 2006; Karl et al. 2006; Santer et al. 2013). In the vertical average, our Phi35 integration produces maximum warming in the tropics, and our Phi35-20 integration produces peak warming in the midlatitudes. So the warming pattern in satellite observations most closely resembles that of our Phi35-20 integration, which, as shown above, produces much more $\mathrm{HC}$ widening than the Phi35 integration. If the warming pattern in satellite observations is correct, this suggests that the $\mathrm{HC}$ might be widening at a rate much faster than in typical simulations of global warming.

There is, however, much uncertainty surrounding satellite observations, due to numerous changes in processing software, the appearance of cooling trends in some datasets, and some trends that appeared to contradict the lapse-rate feedback principle (for extensive discussions, see Karl et al. 2006; Santer et al. 2008; Thorne et al. 2011). While there are quantitative differences between datasets, they all do show enhanced warming in the $\mathrm{NH}$ midlatitudes, with some datasets also showing mildly enhanced warming in the SH midlatitudes (see, e.g., Karl et al. 2006, Fig. 3.5; Santer et al. 2013, Fig. S5). This does not prove that midlatitude amplification is a reality, but it does compel us to consider it as a serious possibility.

In simulations with historical forcings, some comprehensive models do produce midlatitude amplification, but most do not (Santer et al. 2013). The multimodel mean exhibits peak warming that is approximately flat equatorward of $\sim 30^{\circ}$, while observations show a more pronounced local maximum in warming near $30^{\circ}$ (Santer et al. 2013, Fig. S5). This lack of midlatitude warming might cause comprehensive models to underpredict rates of tropical widening. Johanson and Fu (2009) and Allen et al. (2012) have shown results suggesting that comprehensive GCM have indeed underproduced historical tropical widening trends, but Davis and Rosenlof (2012) offer evidence that the observed widening trend may not be robust. So further monitoring and further analysis are needed to determine if there is a real discrepancy between models and observations. But our key point remains: midlatitude amplification is a very real possibility, and it might greatly enhance the rate of tropical widening.

What might be causing enhanced midlatitude warming in the first place? Allen et al. (2012) proposed that such warming may be due to tropospheric ozone or absorbing aerosols, which are more spatially confined than carbon dioxide. Another possibility is that changes in subtropical humidity and cloud cover are contributing to this pattern. These changes may in turn be related to changes in ocean temperatures. For example, Hoerling and Kumar (2003) have shown that, on multiyear time scales, cooling in the eastern tropical Pacific can lead to enhanced warming in the midlatitudes. It is left to future studies to pinpoint the possible drivers of midlatitude warming more conclusively. 


\section{Summary and conclusions}

Using an idealized GCM, we have shown that the contrast between the El Niño and global warming circulation responses depends on the meridional structure of the thermal forcing. A narrow positive forcing centered at the equator produces $\mathrm{HC}$ contraction and an equatorward shift of the jets, while a wider forcing has the opposite effect. Furthermore, warming concentrated in the midlatitudes produces much-amplified $\mathrm{HC}$ expansion and poleward jet shifts when compared to a thermal forcing that is spread over the tropics. These responses are primarily sensitive to changes in the meridional structure of the thermal forcing and are less sensitive to changes in the lapse rate. The exceptionally large circulation response to midlatitude warming points to the possibility that comprehensive GCM might underpredict widening of the tropical belt.

We have also provided a simplified way of understanding these circulation responses. Specifically, we can parameterize the TEM circulation as the meridional diffusion of potential temperature. When a thermal forcing is applied, it results in anomalous diabatic cooling, and hence anomalous TEM descent, on the poleward flank of the thermal forcing. For a narrow (wide) thermal forcing, this anomalous descent occurs on the equatorward (poleward) side of the $\mathrm{HC}$ edge, producing an equatorward (poleward) shift of the $\mathrm{HC}$ edge.

One area ripe for future study concerns the possible causes of amplified warming in the midlatitudes. Possible contributors include absorbing aerosols (Allen et al. 2012) or long-term changes in tropical SST (Hoerling and Kumar 2003). Experiments with full and intermediatecomplexity GCM will be key to testing various hypotheses. Finally, every effort should be made to determine the robustness of the midlatitude amplification patterns shown in satellite observations.

Acknowledgments. We thank Gang Chen, Chris Bretherton, Tiffany Shaw, and Bill Randel for helpful discussions. We also thank Mark Cane for constructive comments on a draft manuscript and Thomas Reichler, Amy Butler, and an anonymous reviewer for valuable feedback on the submitted manuscript. This work was supported by grants of the U.S. National Science Foundation to Columbia University and New York University.

\section{APPENDIX}

\section{GCM Description}

Many aspects of the model we use are identical to those of Tandon et al. (2011), but we provide here the essential details. We use the spectral dynamical core of the Geophysical Fluid Dynamics Laboratory (GFDL) Flexible Modeling System (FMS). The horizontal truncation is T42 for all results presented in the paper, but we have also tested T85 and found no notable differences. The vertical level interfaces, in sigma coordinates, are $\sigma_{i}=(i / L)^{2}, i=0,1,2, \ldots, L$, where $L$ is an integer. For all results presented in the paper $L=40$, but we have also tested $L=80$ and found no notable differences.

We add terms to the temperature equation to capture convective and radiative processes, as well as our imposed thermal forcing. Specifically,

$$
\frac{\partial T}{\partial t}=\ldots-\frac{T-T_{C}}{\tau_{C}}-\frac{T-T_{R}}{\tau_{R}}+\tilde{Q}\left(\frac{p}{p_{0}}\right)^{R / c_{p}},
$$

where $T_{C}$ and $\tau_{C}$ are the convective equilibrium temperature and time scale, respectively; $T_{R}$ and $\tau_{R}$ are the radiative equilibrium temperature and time scale, respectively; $Q$ is our external thermal forcing in terms of potential temperature, given by (1); $R$ is the gas constant for dry air; and $c_{p}$ is the specific heat of dry air. Here, $T_{R}$ and $\tau_{R}$ are exactly as given in Tandon et al. (2011), mimicking the thermal structure of an atmosphere in gray radiative equilibrium.

$$
T_{C} \text { is given by }
$$

$$
\begin{aligned}
& T_{C}(\lambda, \phi, p, t) \\
& \quad= \begin{cases}T_{m}(\lambda, \phi, p, t)-E_{C}(\lambda, \phi, t) & p_{\mathrm{LNB}}(\lambda, \phi, t) \leq p \leq p_{0} \\
T(\lambda, \phi, p, t) & p<p_{\mathrm{LNB}}(\lambda, \phi, t),\end{cases}
\end{aligned}
$$

where

$$
\begin{aligned}
E_{C}(\lambda, \phi, t)= & \frac{1}{p_{\mathrm{LNB}}(\lambda, \phi, t)-p_{0}} \\
& \times \int_{p_{0}}^{p_{\mathrm{LNB}}(\lambda, \phi, t)}\left[T_{m}\left(\lambda, \phi, p^{\prime}, t\right)-T\left(\lambda, \phi, p^{\prime}, t\right)\right] d p^{\prime}
\end{aligned}
$$

ensures conservation of enthalpy in (A2). Then (A2) is applicable only when $E_{C}>0$. If $E_{C} \leq 0$ then convection is inhibited, that is, $T_{C}=T$ in the entire column. $T_{m}$ is the moist adiabat,

$$
T_{m}(\lambda, \phi, p, t)=T_{s}(\lambda, \phi, t)\left(\frac{p}{p_{0}}\right)^{R\left(\Gamma_{m}+\tilde{\Gamma}\right) / g}+\Delta_{z} \log \frac{p}{p_{0}},
$$

where $T_{S}$ is the surface temperature at longitude-latitudetime $(\lambda, \phi, t) ; \Gamma_{m}=6 \mathrm{~K} \mathrm{~km}^{-1} ; \tilde{\Gamma}$ is the lapse rate perturbation given by (2); $\Delta_{z}=7 \mathrm{~K}$; and $p_{\mathrm{LNB}}$ is the level of 
neutral buoyancy for ascent from the surface along $T_{m}$. In contrast to Schneider and Walker (2006) and Tandon et al. (2011), (A4) includes a second term which makes the lapse rate increase with altitude. This produces more realistic alignment between the upper- and lower-level wind maxima. The time scale $\tau_{C}$ is set to $4 \mathrm{~h}$.

There is no topography in this model. For $\sigma>0.7$, winds are linearly damped as in Held and Suarez (1994). We apply $\nabla^{6}$ hyperviscosity, and above $5 \mathrm{hPa}$, we apply a sponge layer top with the same functional form as in Polvani and Kushner (2002).

\section{REFERENCES}

Allen, R. J., S. C. Sherwood, J. R. Norris, and C. S. Zender, 2012: The equilibrium response to idealized thermal forcings in a comprehensive GCM: Implications for recent tropical expansion. Atmos. Chem. Phys., 12, 4795-4816, doi:10.5194/ acp-12-4795-2012.

Butler, A. H., D. W. J. Thompson, and R. Heikes, 2010: The steadystate atmospheric circulation response to climate change-like thermal forcings in a simple general circulation model. J. Climate, 23, 3474-3496.

,-- , and T. Birner, 2011: Isentropic slopes, downgradient eddy fluxes, and the extratropical atmospheric circulation response to tropical tropospheric heating. J. Atmos. Sci., 68, 2292-2305.

Chang, E. K. M., 1995: The influence of Hadley circulation intensity changes on extratropical climate in an idealized model. J. Atmos. Sci., 52, 2006-2024.

Chen, G., R. A. Plumb, and J. Lu, 2010: Sensitivities of zonal mean atmospheric circulation to SST warming in an aqua-planet model. Geophys. Res. Lett., 37, L12701, doi:10.1029/ 2010 GL043473.

_ interaction in the atmospheric circulation response to climate forcing: Uniform SST warming in an idealized aquaplanet model. J. Atmos. Sci., in press.

Davis, S. M., and K. H. Rosenlof, 2012: A multidiagnostic intercomparison of tropical-width time series using reanalyses and satellite observations. J. Climate, 25, 1061-1078.

DiNezio, P. N., A. C. Clement, G. A. Vecchi, B. J. Soden, B. P. Kirtman, and S.-K. Lee, 2009: Climate response of the equatorial Pacific to global warming. J. Climate, 22, 4873-4892.

Edmon, H. J., B. J. Hoskins, and M. E. McIntyre, 1980: EliassenPalm cross sections for the troposphere. J. Atmos. Sci., 37, 2600-2616.

Frierson, D. M. W., I. M. Held, and P. Zurita-Gotor, 2007a: A grayradiation aquaplanet moist GCM. Part II: Energy transports in altered climates. J. Atmos. Sci., 64, 1680-1693.

_ _ J. Lu, and G. Chen, 2007b: Width of the Hadley cell in simple and comprehensive general circulation models. Geophys. Res. Lett., 34, L18804, doi:10.1029/2007GL031115.

Fu, Q., and P. Lin, 2011: Poleward shift of subtropical jets inferred from satellite-observed lower-stratospheric temperatures. J. Climate, 24, 5597-5603.

—, C. M. Johanson, J. M. Wallace, and T. Reichler, 2006: Enhanced mid-latitude tropospheric warming in satellite measurements. Science, 312, 1179, doi:10.1126/science.1125566.

Gastineau, G., H. Le Treut, and L. Li, 2008: Hadley circulation changes under global warming conditions indicated by coupled climate models. Tellus, 60, 863-884, doi:10.1111/ j.1600-0870.2008.00344.x.

Gerber, E. P., and L. M. Polvani, 2009: Stratosphere-troposphere coupling in a relatively simple AGCM: The importance of stratospheric variability. J. Climate, 22, 1920-1933.

— C. Orbe, and L. M. Polvani, 2009: Stratospheric influence on the tropospheric circulation revealed by idealized ensemble forecasts. Geophys. Res. Lett., 36, L24801, doi:10.1029/ 2009GL040913.

Gill, A. E., 1980: Some simple solutions for heat-induced tropical circulation. Quart. J. Roy. Meteor. Soc., 106, 447-462, doi:10.1002/qj.49710644905.

Haigh, J. D., M. Blackburn, and R. Day, 2005: The response of tropospheric circulation to perturbations in lower-stratospheric temperature. J. Climate, 18, 3672-3685.

Held, I. M., 2000: The general circulation of the atmosphere: 2000 program in geophysical fluid dynamics. J.-L. Thiffeault, Ed., Woods Hole Oceanographic Institute Tech. Rep. WHOI2001-03, $181 \mathrm{pp}$.

_ , and A. Y. Hou, 1980: Nonlinear axially symmetric circulations in a nearly inviscid atmosphere. J. Atmos. Sci., 37, 515-533.

_ circulation of the troposphere. Advances in Geophysics, Vol. 28, Academic Press, 3-31.

— , and M. J. Suarez, 1994: A proposal for the intercomparison of the dynamical cores of atmospheric general circulation models. Bull. Amer. Meteor. Soc., 75, 1825-1830.

— , and T. Schneider, 1999: The surface branch of the zonally averaged mass transport circulation in the troposphere. J. Atmos. Sci., 56, 1688-1697.

Hoerling, M., and A. Kumar, 2003: The perfect ocean for drought. Science, 299, 691-694, doi:10.1126/science.1079053.

Johanson, C. M., and Q. Fu, 2009: Hadley cell widening: Model simulations versus observations. J. Climate, 22, 2713-2725.

Kang, S. M., and L. M. Polvani, 2011: The interannual relationship between the latitude of the eddy-driven jet and the edge of the Hadley cell. J. Climate, 24, 563-568.

— D. M. W. Frierson, and I. M. Held, 2009: The tropical response to extratropical thermal forcing in an idealized GCM: The importance of radiative feedbacks and convective parameterization. J. Atmos. Sci., 66, 2812-2827.

Karl, T. R., S. J. Hassol, C. D. Miller, and W. L. Murray, Eds., 2006: Temperature Trends in the Lower Atmosphere: Steps for Understanding and Reconciling Differences. U.S. Climate Change Science Program/Subcommittee on Global Change Research, 164 pp.

Kidston, J., G. K. Vallis, S. M. Dean, and J. A. Renwick, 2010: Can the increase in the eddy length scale under global warming cause the poleward shift of the jet streams? J. Climate, 24, 3764-3780.

Levine, X. J., and T. Schneider, 2011: Response of the Hadley circulation to climate change in an aquaplanet GCM coupled to a simple representation of ocean heat transport. J. Atmos. Sci., 68, 769-783.

Lorenz, D. J., and E. T. DeWeaver, 2007: Tropopause height and zonal wind response to global warming in the IPCC scenario integrations. J. Geophys. Res., 112, D10119, doi:10.1029/ 2006JD008087.

Lu, J., G. Chen, and D. M. W. Frierson, 2008: Response of the zonal mean atmospheric circulation to El Niño versus global warming. J. Climate, 21, 5835-5851.

,-- , and,- 2010 : The position of the midlatitude storm track and eddy-driven westerlies in aquaplanet AGCMs. J. Atmos. Sci., 67, 3984-4000. 
Miller, R. L., G. A. Schmidt, and D. T. Shindell, 2006: Forced annular variations in the 20th century Intergovernmental Panel on Climate Change Fourth Assessment Report models. J. Geophys. Res., 111, D18101, doi:10.1029/2005JD006323.

Peixoto, J. P., and A. H. Oort, 1992: Physics of Climate. American Institute of Physics, 520 pp.

Phillips, N., 1954: Energy transformations and meridional circulations associated with simple baroclinic waves in a two-level, quasi-geostrophic model. Tellus, 6, 273-286, doi:10.1111/j.2153-3490.1954.tb01123.x.

Polvani, L. M., and P. J. Kushner, 2002: Tropospheric response to stratospheric perturbations in a relatively simple general circulation model. Geophys. Res. Lett., 29, 1114, doi:10.1029/ 2001 GL014284.

Rivière, G., 2011: A dynamical interpretation of the poleward shift of the jet streams in global warming scenarios. J. Atmos. Sci., 68, 1253-1272.

Robinson, W. A., 2002: On the midlatitude thermal response to tropical warmth. Geophys. Res. Lett., 29, 1190, doi:10.1029/ 2001GL014158.

Santer, B. D., and Coauthors, 2003: Behavior of tropopause height and atmospheric temperature in models, reanalyses, and observations: Decadal changes. J. Geophys. Res., 108, 4002, doi:10.1029/2002JD002258.

__ , and Coauthors, 2008: Consistency of modelled and observed temperature trends in the tropical troposphere. Int. J. Climatol., 28, 1703-1722, doi:10.1002/joc.1756.

— mospheric temperature. Proc. Natl. Acad. Sci. USA, 110, 2633, doi:10.1073/pnas.1210514109.

Schneider, E. K., and R. S. Lindzen, 1976: The influence of stable stratification on the thermally driven tropical boundary layer. J. Atmos. Sci., 33, 1301-1307.

Schneider, T., 2004: The tropopause and the thermal stratification in the extratropics of a dry atmosphere. J. Atmos. Sci., 61, 1317-1340.

_ , and C. C. Walker, 2006: Self-organization of atmospheric macroturbulence into critical states of weak nonlinear eddyeddy interactions. J. Atmos. Sci., 63, 1569-1586.

Seager, R., N. Harnik, Y. Kushnir, W. Robinson, and J. Miller, 2003: Mechanisms of hemispherically symmetric climate variability. J. Climate, 16, 2960-2978.
Seidel, D. J., Q. Fu, W. J. Randel, and T. J. Reichler, 2008: Widening of the tropical belt in a changing climate. Nat. Geosci., 1, 21-24, doi:10.1038/ngeo.2007.38.

Sobel, A. H., J. Nilsson, and L. M. Polvani, 2001: The weak temperature gradient approximation and balanced tropical moisture waves. J. Atmos. Sci., 58, 3650-3665.

Soden, B. J., R. T. Wetherald, G. L. Stenchikov, and A. Robock, 2002: Global cooling after the eruption of Mount Pinatubo: A test of climate feedback by water vapor. Science, 296, 727-730, doi:10.1126/science.296.5568.727.

Son, S.-W., and S. Lee, 2005: The response of westerly jets to thermal driving in a primitive equation model. J. Atmos. Sci., 62, 3741-3757.

Tandon, N. F., L. M. Polvani, and S. M. Davis, 2011: The response of the tropospheric circulation to water vapor-like forcings in the stratosphere. J. Climate, 24, 5713-5720.

Thorne, P. W., J. R. Lanzante, T. C. Peterson, D. J. Seidel, and K. P. Shine, 2011: Tropospheric temperature trends: History of an ongoing controversy. Wiley Interdiscip. Rev. Climate Change, 2, 66-88, doi:10.1002/wcc.80.

Walker, C. C., and T. Schneider, 2006: Eddy influences on Hadley circulations: Simulations with an idealized GCM. J. Atmos. Sci., 63, 3333-3350.

Wang, B., and T. Li, 1993: A simple tropical atmosphere model of relevance to short-term climate variations. J. Atmos. Sci., 50, 260-284.

Wang, S., E. P. Gerber, and L. M. Polvani, 2012: Abrupt circulation responses to tropical upper-tropospheric warming in a relatively simple stratosphere-resolving AGCM. J. Climate, 25, 4097-4115.

World Meteorological Organization, 1957: Meteorology-A threedimensional science. WMO Bull., 4, 134-138.

Wu, Y., M. Ting, R. Seager, H.-P. Huang, and M. Cane, 2011: Changes in storm tracks and energy transports in a warmer climate simulated by the GFDL CM2.1 model. Climate Dyn., 37, 53-72, doi:10.1007/s00382-010-0776-4

— R. Seager, M. Ting, N. Naik, and T. A. Shaw, 2012: Atmospheric circulation response to an instantaneous doubling of carbon dioxide. Part I: Model experiments and transient thermal response in the troposphere. J. Climate, 25, 2862-2879.

Yin, J. H., 2005: A consistent poleward shift of the storm tracks in simulations of 21st century climate. Geophys. Res. Lett., 32, L18701, doi:10.1029/2005GL023684. 